\title{
On the Contribution of Higher Azimuthal Modes to the Near- and Far-Field of Jet Mixing Noise
}

\author{
A. Neifeld* and R. Ewert ${ }^{\dagger}$ \\ Institute of Aerodynamics and Flow Technology, Technical Acoustics Branch, \\ German Aerospace Center (DLR), Lilienthalplatz 7, 38108 Braunschweig, Germany
}

\begin{abstract}
The prediction of jet mixing noise is studied using a stochastic realization of the Tam \& Auriault source model. The acoustical sources are generated by means of the $R$ andom Particle-Mesh Method (RPM), which utilizes turbulence statistics as provided by solutions to the Reynolds Averaged Navier-Stokes (RANS) equations. The generated stochastic sound sources closely realize the two-point cross-correlation function used in the jet noise model to prescribe the fine-scale sound source. The RPM code is coupled with the DLR CAA solver PIANO. The azimuthal-modal decomposed linearized Euler equations are applied as governing equations. With this approach, it is possible to evaluate jet noise spectra at any position in the near-field. Based on an azimuthal decomposition, 3-D sound radiation from the jet can be reproduced at the computational price of a few axisymmetric 2-D computations. Furthermore, it will be shown, that we are able to verify the implemented methodology with the results published for the genuine model. The spectra are correctly predicted in terms of sound pressure levels, Mach scaling exponent and spectral shape. A Strouhal number range of up to $S t=10$ can be covered using the first six azimuthal mode components of the broadband source. To reach higher Strouhal numbers more azimuthal modes have to be adopted. The presented results reveal the importance of individual azimuthal contributions to the total spectra. To evaluate the spectra in the far-field, the generated near-field noise is extrapolated with a modal Ffowcs-Williams \& Hawkings (FWH) method. For the static single stream jet $(M a=0.9)$ two different kinds of extrapolation were used - a simplified extrapolation and the modal FWH method. With this computational case, it was possible to predict a jet noise spectrum in the range of $S t=0.01 \ldots 20$. To investigate the effect of different nozzle configurations on sound generation, different nozzle configurations, i.e. dual-stream nozzles with and without nozzle lip treatments are simulated. Good agreement with experimental data for the noise reduction potential of nozzle lip treatments is found.
\end{abstract}

\section{Introduction}

In recent years, reasonable achievements have been reached in the numerical simulation of jet noise. Especially, high fidelity methods (DNS, LES/DES and derivatives of it) are utilized for scale resolving simulation of increasingly complex flow problems to understand and analyze the sound generation mechanisms of a jet. But, there is also a need for fast prediction tools, which allow to study the effect of design changes on jet noise in an industrial environment. The stochastic source modeling studied in this paper aims at approaching this goal through a significantly reduced numerical simulation time compared to scale resolving simulation. For the simulation of the sound generation and radiation problem, the governing linearized Euler equations (LEE) are forced with stochastic sound sources. The approach can be deemed to be an alternative way to solve acoustic analogy or equivalent sound source models based on the modeling of source cross-covariances (sometimes referred to as semi-empirical source models). Scaling of the main parameters of the cross-covariance models can be derived from steady RANS simulation. The solution of established semi-empirical jet noise models with state of the art CAA tools opens the encouraging perspective to enable the solution of all major models in a unified simulation environment (Statistical Noise Toolbox).

\footnotetext{
*Research Scientist, AIAA Member, andrej.neifeld@dlr.de

†Senior Scientist DLR, Senior AIAA Member, roland.ewert@dlr.de
} 
For a high numerical efficiency of a stochastic jet source model, we promote an azimuthal Fourier series decomposition of a full 3-D jet noise problem into a set of axisymmetric modal problems. Hence, instead of resolving the azimuthal direction a limited number of axisymmetric simulations for each azimuthal mode have to be realized to obtain a solution to the complete $3-\mathrm{D}$ problem. The idea is to simulate the azimuthal modes independently from each other and merge them to a single solution in the postprocessing. The advantage of such an approach is the drastically reduced number of grid points in comparison to the fully resolved problem. But also, in respect to increasing frequency resolution, the growth of grid points in modal approach appears with the power of two instead of three.

We are pursuing in the ongoing work the goal to verify our methodology with the jet configurations applied in the work of Tam \& Auriault (T\&A). ${ }^{1}$ The modeling of sound sources in RPM is closely related to the original model, but due to the azimuthal-modal decomposition, the RPM realization has some additional features (refer to Ewert et al. ${ }^{5}$ ). For example, the genuine T\&A model is solved in the frequency domain, therefore it needs individual (adjoint) CAA compuations for each observer position and frequency band. A broadband stochastic method solved in the time-domain for each azimuthal mode order needs only about six axisymmetric, i.e. quasi 2-D computations to reach a jet Strouhal number of $S t=10$. Furthermore, the latter apporach can be applied to spreading (i.e. non-parallel) jets without increased computational effort.

As it will be shown in Sec.V, the results are encouraging. In all acoustical aspects the comparison between presented results and the genuine T\&A model shows considerably good match. The analyzed spectra are evaluated and compared in the polar angle range proposed by the T\&A model for the fine scale jet noise. Regarding the meaning of each azimuthal mode spectrum to the total spectrum, it was found that every of them have a certain contribution to the total spectrum. In the low frequency range, the azimuthal modes higher than $m=0$ have hardly any influence. But, since low azimuthal mode contributions fall off rapidly at higher Strouhal numbers, the higher modes gain their contribution and become even stronger than the contribution of the zeroth azimuthal mode in this wave number regime.

\section{Governing Equations}

The Linearized Euler equations will be used in this work as governing equations. A complex-valued azimuthal-modal formulation of these equations is implemented in PIANO and used here for jet noise computations. The mean flow and the acoustic quantities $\rho^{0}, \mathbf{u}^{0}, p^{0}, \rho^{\prime}, \mathbf{u}^{\prime}, p^{\prime}$, can be expanded as complex Fourier series. Here, the velocity components are given in cylindrical coordinates, e.g. $\mathbf{u}^{0}=\left(u_{x}^{0}, u_{r}^{0}, u_{\phi}^{0}\right)^{T}$ and $\mathbf{u}^{\prime}=\left(u_{x}^{\prime}, u_{r}^{\prime}, u_{\phi}^{\prime}\right)^{T}$. All variables are Fourier transformed in azimuthal direction, i.e.

$$
b(x, r, \phi, t)=\sum_{m=-\infty}^{\infty} \hat{b}_{m}(x, r, t) \exp (\mathrm{i} m \phi) \quad \hat{b}_{m}=b_{m \Re}+\mathrm{i} b_{m \Im} \in \mathbb{C},
$$

where $b$ is a placeholder for the actually considered variable and $\hat{b}_{m}$ represents its complex-valued related Fourier transform of mode order $m$. The perturbation quantities are decomposed to more than one azimuthal mode, however, at the moment, for the sake of simplicity, only the zeroth azimuthal mode $(\mathrm{m}=0)$ is considered for the mean flow. For nozzle geometries with azimuthally invariant geometry the mean-flow will have only a non-zero contribution to the zeroth mode, hence this procedure is exact. For computations with complex nozzle geometries, e.g. nozzles with serrations or chevrons, also higher azimuthal mode components are present, which therefore are neglected in the current procedure. This implicitly means that for complex nozzle geometries the mean-flow refraction effects will be approximately resolved in regions of high azimuthal mean-flow variation, i.e. immediately downstream of the nozzle exit. In Sec. V the error that stems from this simplification will be investigated thoroughly with a serrated nozzle test cases that reveals only a relative small impact on the prediction of refraction effects in the jet flow. ${ }^{\text {a }}$ Furthermore, we will restrict our analysis to flows without swirl, i.e. $u_{\phi}^{0}=0$.

The modal decomposed governing equations read

$$
\frac{\partial \hat{\mathbf{q}}_{m}}{\partial t}+\hat{\mathbf{A}}_{m} \frac{\partial \hat{\mathbf{q}}_{m}}{\partial x}+\hat{\mathbf{B}}_{m} \frac{\partial \hat{\mathbf{q}}_{m}}{\partial r}+\hat{\mathbf{C}}_{m} \frac{\hat{\mathbf{q}}_{m}}{r}+\hat{\mathbf{D}}_{m} \hat{\mathbf{q}}_{m}=\hat{\mathbf{s}}_{m}
$$

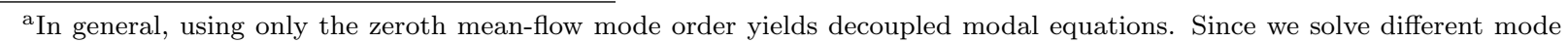
order simultaneously within one computation, in principle an extension to include also higher mean-flow mode contributions is possible. 
with

$$
\begin{gathered}
\hat{\mathbf{q}}_{m}=\left(\begin{array}{c}
\hat{\rho}_{m}^{\prime} \\
\hat{u}_{x, m}^{\prime} \\
\hat{u}_{r, m}^{\prime} \\
\hat{u}_{\phi, m}^{\prime} \\
\hat{p}_{m}^{\prime}
\end{array}\right) \quad \hat{\mathbf{s}}_{m}=\left(\begin{array}{c}
0 \\
0 \\
0 \\
0 \\
\hat{\theta}_{m}
\end{array}\right) \quad \hat{\mathbf{A}}_{m}=\left(\begin{array}{ccccc}
u_{x}^{0} & \rho^{0} & 0 & 0 & 0 \\
0 & u_{x}^{0} & 0 & 0 & \frac{1}{\rho^{0}} \\
0 & 0 & u_{x}^{0} & 0 & 0 \\
0 & 0 & 0 & u_{x}^{0} & 0 \\
0 & \gamma p^{0} & 0 & 0 & u_{x}^{0}
\end{array}\right) \quad \hat{\mathbf{B}}_{m}=\left(\begin{array}{ccccc}
u_{r}^{0} & 0 & \rho^{0} & 0 & 0 \\
0 & u_{r}^{0} & 0 & 0 & 0 \\
0 & 0 & u_{r}^{0} & 0 & \frac{1}{\rho^{0}} \\
0 & 0 & 0 & u_{r}^{0} & 0 \\
0 & 0 & \gamma p^{0} & 0 & u_{r}^{0}
\end{array}\right) \\
\hat{\mathbf{C}}_{m}=\left(\begin{array}{ccccc}
u_{r}^{0} & 0 & \rho^{0} & -i m \rho^{0} & 0 \\
0 & 0 & 0 & 0 & 0 \\
0 & 0 & 0 & 0 & 0 \\
0 & 0 & 0 & u_{r}^{0} & -\mathrm{i} \frac{m}{\rho^{0}} \\
0 & 0 & \gamma p^{0} & -\mathrm{i} m & \gamma u_{r}^{0}
\end{array}\right) \hat{\mathbf{D}}_{m}=\left(\begin{array}{ccccc}
\frac{\partial u_{x}^{0}}{\partial x}+\frac{\partial u_{r}^{0}}{\partial r} & \frac{\partial \rho^{0}}{\partial x^{0}} & \frac{\partial \rho^{0}}{\partial r} & 0 & 0 \\
-\frac{1}{\left(\rho^{0}\right)^{2}} \frac{\partial p^{0}}{\partial x} & \frac{\partial u_{x}}{\partial x} & \frac{\partial u_{x}^{0}}{\partial r} & 0 & 0 \\
-\frac{1}{\left(\rho^{0}\right)^{2}} \frac{\partial p^{0}}{\partial r} & \frac{\partial u_{r}^{0}}{\partial x} & \frac{\partial u_{r}^{0}}{\partial r} & 0 & 0 \\
0 & 0 & 0 & 0 \\
0 & \frac{\partial p^{0}}{\partial x} & \frac{\partial p^{0}}{\partial r} & 0 & \gamma\left(\frac{\partial u_{x}^{0}}{\partial x}+\frac{\partial u_{r}^{0}}{\partial r}\right)
\end{array}\right)
\end{gathered}
$$

The complex-valued vector $\hat{\mathbf{s}}_{m}$ establishes the right hand side forcing of the governing equations with a single non-zero component $\hat{\theta}_{m}$ on the right-hand side of the pressure equation. More details of the modal source $\hat{\theta}_{m}$ are described in the next section. The azimuthal mode order $m$ appears at three positions in the matrix $\hat{\mathbf{C}}_{m}$, i.e. in the density, the $\phi$-momentum and the pressure equation, respectively.

In the first place, our aim is to analyze only the fine scale noise of a jet. Therefore, we neglect the meanflow gradient terms in Eqn. (2). Hence, the triggering of hydrodynamic instabilities, which are believed to be the main reason for large scale noise generation, is suppressed and only fine scale noise generation at polar angles mainly perpendicular to the jet axis is studied in this work. The large scale noise is planed to be investigated in more detail in further work.

\section{RPM sources}

\section{A. Basic idea of stochastic source modeling by RPM}

The modal sound sources are realized with the Random Particle-Mesh Method (RPM) as a complex-valued quantity, which appears in the linearized Euler equations on the right hand side of the pressure equation (see Eqn.(2)). For more details about the stochastic source modeling with the RPM method refer to Ewert. ${ }^{3}$ The stochastic realization of the Tam \& Auriault source term and its azimuthal decomposition was discussed in more details in Ref. ${ }^{5}$ A brief overview will be given subsequently as it concerns the discussion in this paper. In 3-D the source term of the the Tam \& Auriault jet mixing noise model is a scalar quantity $\theta$ on the right-hand side of the pressure equation. This source term is the substantial time derivative of a quantity $q_{s}$ introduced by Tam \& Auriault ${ }^{1}$ as a variable loosely related to turbulent pressure fluctuations, i.e.

$$
\theta \equiv \frac{D q_{s}}{D t}
$$

The two-point space-time correlation between point positions 1 and 2 of the source term are modeled by

$$
\left\langle\theta_{1} \theta_{2}\right\rangle=\hat{R} \times \exp \left\{-\frac{|\xi|}{u_{j} \tau_{s}}-\frac{\ln 2}{l_{s}^{2}}\left[\left(\xi-u_{j} \tau\right)^{2}+\eta^{2}+\zeta^{2}\right]\right\}
$$

The variables $\xi, \eta$ and $\zeta$ denote here the relative spatial difference between two source positions, $u_{j}$ denotes the convective velocity in the source domain and $\hat{R}$ represents the source variance

$$
\hat{R}=\frac{\hat{q}_{s}^{2}}{c^{2} \tau_{s}^{2}} .
$$

In the Tam \& Auriault model the parameters on the right-hand side are specified by ${ }^{1}$

$$
\frac{\hat{q}_{s}}{c}=\frac{2}{3} A \rho^{0} k
$$

with $A=0.755$ and $k$ denotes the turbulent kinetic energy from RANS. The parameters $\tau_{s}$ and $l_{s}$ are the time- and length-scale, respectively. The quantities $\hat{R}, \tau_{s}, l_{s}, u_{j}$ can be obtained from a RANS solution 
utilizing a two-equation/Reynold Stress turbulence model (refer to Fig. 1). The length-scale infers from a $k-\epsilon$ model via

$$
l_{s}=c_{l} \frac{k^{3 / 2}}{\epsilon}
$$

with $c_{l}=0.256$. The time-scale is inferred from

$$
\tau_{s}=c_{\tau} \frac{k}{\epsilon}
$$

where $c_{\tau}=0.233$. The implementation of the above model in RPM varies slightly, i.e. instead of Eqn. (4) the following two-point correlations are realized by the stochastically generated fluctuations $\theta$,

$$
\left\langle\theta_{1} \theta_{2}\right\rangle=\hat{R}^{*} \times \exp \left\{-\frac{|\tau|}{\tau_{s}}-\frac{\pi}{4 l_{s}^{* 2}}\left[\left(\xi-u_{j} \tau\right)^{2}+\eta^{2}+\zeta^{2}\right]\right\},
$$

with the term different indicated by the box. This term leads to Helmholtz similar spectra instead of Strouhal similar spectra that follow from the model given by Eq. (4). To correct this deviation, we found that it is possible to use a modified length scale coefficient $c_{l}^{*}$ to generated spectra with the desired Strouhal similarity. However, the variance is affected by this correction as well, which can be compensated for by multiplying it with $M a^{3}$. To summarize, the effective length scale parameter and source variance, in the following indicated by an asterisk, which are used in the RPM method to model fluctuations that obey correlations described by the model Eq. (9), are

$$
c_{l}^{*}=\sqrt{\frac{\pi}{4 \ln (2)}} \frac{c_{l}}{M a} \quad \hat{R}^{*}=M a^{3} \hat{R} .
$$

From the length scale parameter the length scale infers as

$$
l_{s}^{*}=c_{l}^{*} \frac{k^{3 / 2}}{\epsilon}
$$

After this correction, the spectra, plotted over frequency, show the expected frequency shift with respect to Mach number variation.

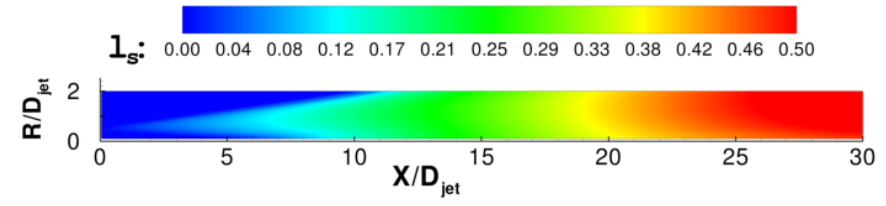

(a) Length scale $l_{s}$

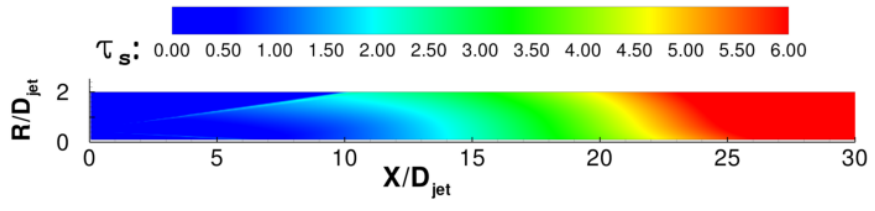

(b) Time scale $\tau_{s}$

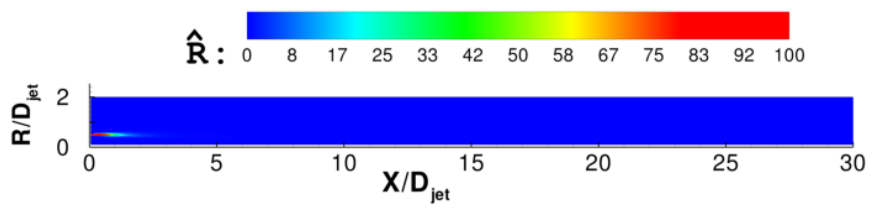

(c) Source variance $\hat{R}$

Figure 1. Statistical quantities extracted from RANS in the defined RPM source domain 
The scalar source term $\theta$ is realized stochastically with the RPM method. ${ }^{4}$ It is obtained by convolving spatiotemporal convective white-noise $\mathcal{U}$ with a filter kernel,

$$
\theta(\boldsymbol{x}, t)=\int A(\boldsymbol{x}) \exp \left(-\frac{\pi}{2 l_{s}^{* 2}}\left(\boldsymbol{x}-\boldsymbol{x}^{\prime}\right)^{2}\right) \mathcal{U}\left(\boldsymbol{x}^{\prime}, t\right) \mathrm{d}^{N} x^{\prime}
$$

In this expression $N$ indicates the dimension of the problem. The scaling function $A$ realizes the desired variance of $\theta$. Here it is assumed to be a function of $\boldsymbol{x}$. The convective white-noise is defined by ${ }^{4}$

$$
\begin{aligned}
\langle\mathcal{U}(\boldsymbol{x}, t)\rangle & =0 \\
\langle\mathcal{U}(\boldsymbol{x}, t) \mathcal{U}(\boldsymbol{x}+\boldsymbol{r}, t)\rangle & =\rho^{0}(\boldsymbol{x})^{-1} \delta(\boldsymbol{r}) \\
\frac{D_{j}}{D t} \mathcal{U} & =-\frac{1}{\tau_{s}} \mathcal{U}+\sqrt{\frac{2}{\tau_{s}}} \xi,
\end{aligned}
$$

where $D_{j} / D t$ denotes the substantial time derivative based on the convection velocity $\boldsymbol{u}_{j}$ and $\xi$ in Eq. (15) denotes a spatio-temporal white-noise source, defined by ${ }^{4}$

$$
\begin{aligned}
\langle\xi(\boldsymbol{x}, t)\rangle & =0, \\
\langle\xi(\boldsymbol{x}, t) \xi(\boldsymbol{x}+\Delta \boldsymbol{x}, t+\Delta t)\rangle & =\rho_{0}(\boldsymbol{x})^{-1} \delta\left(\Delta \boldsymbol{x}-\boldsymbol{u}_{j} \Delta t\right) \delta(\Delta t) .
\end{aligned}
$$

Here $\boldsymbol{x}$ depends on the dimension of the realization, i.e. $\boldsymbol{x} \in \mathbb{R}^{N}$. Since the convective white-noise field is a function of space and time, subsequently we will indicate the convective white-noise fields and the sources generated thereof as $N+1-\mathrm{D}$ realizations to indicate the spatial dimension of the realization $(N$ spatial plus 1 temporal dimension), to indicate the number of spatial dimension considered for its realization. From the model the cross-correlation Eq. (9) derives. Using a scaling

$$
A=\sqrt{\frac{\rho^{0} \hat{R}^{*}}{\left(l_{s}^{*}\right)^{N}}}
$$

with $\hat{R}^{*}$ as given by Eq. (10), the variance corresponds to that used in Eq. (4).

A consistent numerical discretization of the previous procedure leads to a numerical realization with 'random particles' (i.e. convecting particles with associated random variables). Within the limits of accuracy of the numerical approach the particle model provides fluctuations which possess the derived cross-correlations as described by Eq. (9). The filter integral Eq. (12) becomes the weighted sum over a set of $N_{P}$ random particles, i.e.

$$
\theta(\boldsymbol{x}, t) \simeq \sum_{k=1}^{N_{P}} A(\boldsymbol{x}) G\left(\boldsymbol{x}-\boldsymbol{x}_{k}^{c}(t)\right) \frac{r_{k}(t)}{\rho_{0}\left(\boldsymbol{x}_{k}^{c}\right)} .
$$

To each particle a random variable $r_{k}$ is assigned, where the index denotes the particle number. The random particles are convecting in a given velocity field defining the local convection velocity $\boldsymbol{u}_{j}$ (e.g. derived from the steady RANS mean-flow). The actual position of particle $k$ is denoted above by $\boldsymbol{x}_{k}^{c}(t)$ and changes over time. Filter amplitude $A$ and spatial filter are directly taken from integral Eq. (12). For example, the filter kernel in the previous formula is defined by a Gaussian, i.e.

$$
G\left(\boldsymbol{x}-\boldsymbol{x}_{k}^{c}(t)\right)=\exp \left(-\frac{\pi\left(\boldsymbol{x}-\boldsymbol{x}_{k}^{c}\right)^{2}}{2 l_{s}^{* 2}}\right) .
$$

The random particles represent the aforementioned convective white-noise field over a representative control volume surrounding each particle. From the definition of convective white-noise the properties of the random variables are inferred, ${ }^{4}$

$$
\begin{aligned}
\left\langle r_{k}(t)\right\rangle & =0 \\
\left\langle r_{k}(t) r_{l}(t)\right\rangle & =\delta_{k l} \delta m_{k} \\
\dot{r}_{k} & =-\frac{1}{\tau_{s}} r_{k}+\sqrt{\frac{2}{\tau_{s}}} s_{k} \\
\dot{\boldsymbol{x}}_{k}^{c} & =\boldsymbol{u}_{j}\left(\boldsymbol{x}_{k}^{c}\right) .
\end{aligned}
$$


In plain words, the random variables $r_{k}$ are mutually uncorrelated with vanishing mean and a constant variance proportional to $\delta m_{k}$, which is the fluid mass encompassed by the control volume surrounding each particle. The particle drifts with the local convection velocity $\boldsymbol{u}_{j}$ at the particle position $\boldsymbol{x}_{k}^{c}$. The correlations of a random variate associated to a specific particle exhibits an exponential time decay described by a Langevin equation Eq. (23). There, a (temporal) white-noise source term $s_{k}$ appears on the right-hand side, with properties

$$
\begin{aligned}
\left\langle s_{k}(t)\right\rangle & =0 \\
\left\langle s_{k}(t) s_{l}(t+\tau)\right\rangle & =\delta m_{k} \delta(\tau) \delta_{k l} .
\end{aligned}
$$

In other words, $s_{k}$ represents (temporal) white-noise scaled with a factor of magnitude $\delta m_{k}$. The solution of the Langevin equation Eq. (23) with a source having correlation Eq. (26) yields a correlation of variable $r_{k}$

$$
\left\langle r_{k}(t) r_{j l}(t+\tau)\right\rangle=\delta m_{k} \delta_{k l} \exp \left(-\frac{|\tau|}{\tau_{s}}\right) .
$$

The Langevin equation (23) can be solved numerically by the finite-difference equation, see e.g. Pope ${ }^{2}$ pp. 484 ,

$$
r_{k}(t+\Delta t)=\left(1-\frac{\Delta t}{\tau_{s}}\right) r_{k}(t)+\left(\frac{2 \delta m_{k} \Delta t}{\tau_{s}}\right)^{1 / 2} \sigma_{k}(t),
$$

where $\sigma_{k}(t)$ are $N$ mutually uncorrelated standardized Gaussian random variables $\left(\left\langle\sigma_{k}(t)\right\rangle=0,\left\langle\sigma_{k}(t) \sigma_{l}(t)\right\rangle=\right.$ $\left.\delta_{k l}\right)$ which are independent of themselves at different times $\left(\left\langle\sigma_{k}(t) \sigma_{k}\left(t^{\prime}\right)\right\rangle=0\right.$, for $\left.t^{\prime} \neq t\right)$, and which are independent of $r_{k}(t)$ at past times (e.g., $\left\langle\sigma_{k}(t) r_{k}\left(t^{\prime}\right)\right\rangle=0$ for $\left.t^{\prime} \leq t\right)$.

Following this procedure, a source distribution for a cold single stream jet as depicted in Fig. 2 is generated.

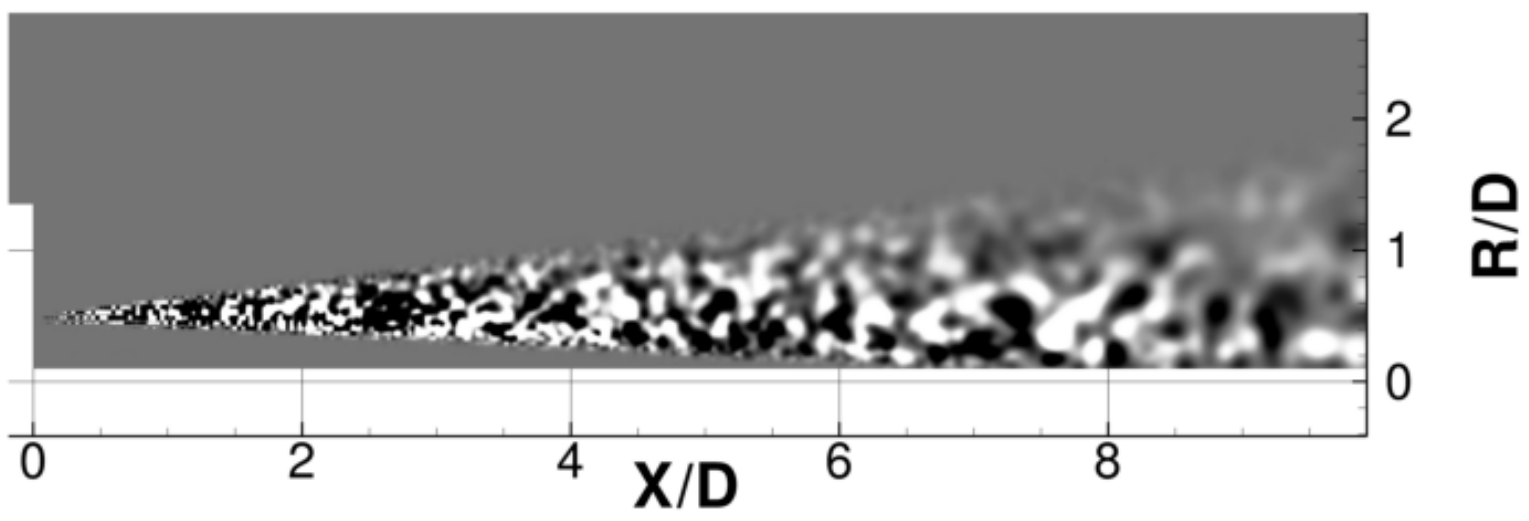

Figure 2. Contour plot of distribution of RPM source term $\theta$ in the shear layer of a jet

\section{B. Azimuthal decomposed jet noise RPM sources}

The complex-valued modal source term of moder order $m$ follows formally from the transformation

$$
\hat{\theta}_{m}(x, r, t)=\frac{1}{2 \pi} \int_{-\pi}^{\pi} \theta(x, r, \phi, t) \exp (-i m \phi) \mathrm{d} \phi .
$$

As was shown in Ref., ${ }^{5}$ the azimuthal Fourier decomposition applied to the $3+1-\mathrm{D}$ RPM source term Eq. (12) yields an realization of the modal source term from a slightly modified $2+1-\mathrm{D}$ RPM procedure. That is, the real and imaginary part of the modal source term are realized by mutually uncorrelated 2+1-D RPM 
realizations each. That is, in this approach the 3-D filter integral Eq. (12) becomes a 2-D integral over the x-r-plane of the jet,

$$
\hat{\theta}_{m}(\boldsymbol{x}, t)=\iint A(x, r) \hat{G}_{m}\left(\frac{r}{l_{s}^{*}}, \frac{r^{\prime}}{l_{s}^{*}}\right) \exp \left(-\frac{\pi\left(x-x^{\prime}\right)^{2}}{2 l_{s}^{* 2}}\right) \hat{\mathcal{U}}\left(x^{\prime}, r^{\prime}, t\right) \mathrm{d} x^{\prime} \mathrm{d} r^{\prime}
$$

Here the real and imaginary part of $\hat{\mathcal{U}}\left(x^{\prime}, r^{\prime}, t\right)$ designate each a real-valued convective white-noise field as defined by Eqs. (13-15) for a 2+1-D realization. Realizations for different mode orders are mutually uncorrelated. The specific modal effect shows up in the real-valued radial filter kernel $\hat{G}_{m}$, which differs between different mode orders. Its derivation was presented in Ref. ${ }^{5}$

The derivation in Ref. ${ }^{5}$ was related to azimuthally invariant nozzle geometries, for which the amplitude function $A$ does not depend on the azimuthal angle. The amplitude function, which relies on RANS statistics via Eq. (18), however, will exhibit for nozzles with lip treatment also refered as noise guide elements (NGEs) an azimuthal dependence, $A(x, r, \phi)$, which provides a modification in the procedure as outlined in Ref. ${ }^{5}$ Specifically, the previous quasi 2-D filter integral changes to

$$
\hat{\theta}_{m}(\boldsymbol{x}, t)=\iint \hat{\mathcal{C}}_{m} \exp \left(-\frac{\pi\left(x-x^{\prime}\right)^{2}}{2 l_{s}^{* 2}}\right) \hat{\mathcal{U}}\left(x^{\prime}, r^{\prime}, t\right) \mathrm{d} x^{\prime} \mathrm{d} r^{\prime},
$$

with a new introduced quantity, which is defined by the discrete convolution of the azimuthally Fourier transformed amplitude function $\hat{A}_{m}$ and the modal filter kernel, i.e.

$$
\hat{\mathcal{C}}_{m}=\sum \hat{A}_{n} \hat{G}_{m-n} .
$$

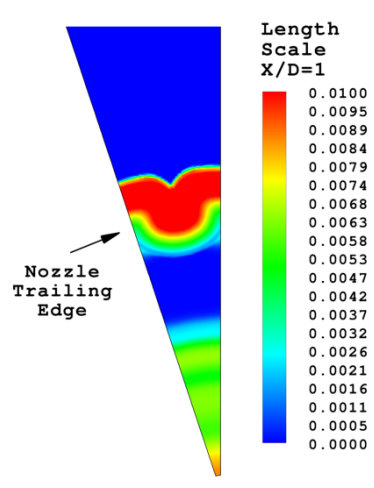

(a) Length scale $l_{s}$

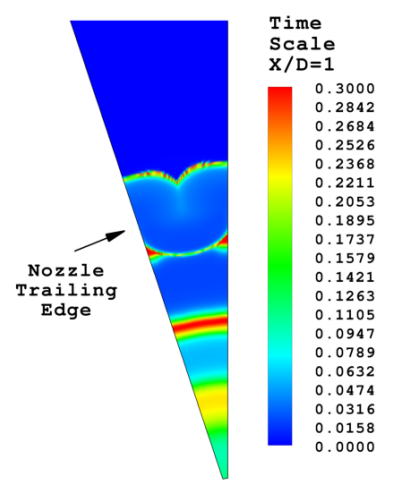

(b) Time scale $\tau_{s}$

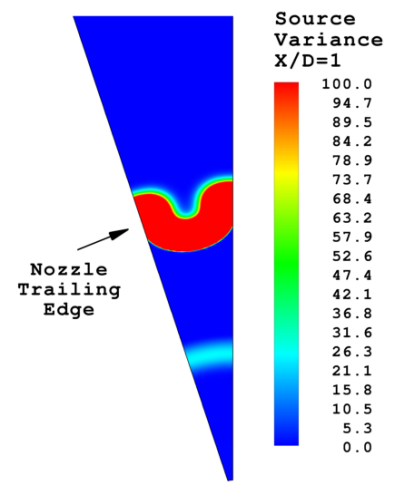

(c) Source variance $\hat{R}$

Figure 3. Statistical quantities of a nozzle with nozzle guide elements extracted from RANS, variation in azimuthal direction at $X / D_{j}=1.0$

In the case of azimuthal uniform axisymmetric nozzles, the mean-flow is represented just by the zeroth azimuthal mode, i.e. only the source variance $\hat{A}_{0}$ has to be considered (which corresponds to A). All other combinations of $\hat{A}_{n} \hat{G}_{n-m},(n \neq 0)$ become zero. Regarding nonuniform azimuthal nozzle geometries, e.g. with serrations or chevrons, the next higher non-zero mean-flow mode is defined by the number of nozzle elements. For example, if a nozzle has 20 serrations the next higher azimuthal mode after the mode $m=0$ would be $m=20$, followed by $m=40$ as the further next higher mode. Howerver, no modes in between are present. Accordingly, Eq. (32) yields for the overall $m=0$ contribution

$$
\hat{\mathcal{C}}_{0}=\ldots+\underbrace{\hat{A}_{-40} \hat{G}_{40}}_{\rightarrow 0}+\underbrace{\hat{A}_{-20} \hat{G}_{20}}_{\rightarrow 0}+\hat{A}_{0} \hat{G}_{0}+\underbrace{\hat{A}_{20} \hat{G}_{-20}}_{\rightarrow 0}+\underbrace{\hat{A}_{40} \hat{G}_{-40}}_{\rightarrow 0}+\ldots
$$

Correspondingly, for the first mode order it follows

$$
\hat{\mathcal{C}}_{1}=\ldots+\underbrace{\hat{A}_{-40} \hat{G}_{41}}_{\rightarrow 0}+\underbrace{\hat{A}_{-20} \hat{G}_{21}}_{\rightarrow 0}+\hat{A}_{0} \hat{G}_{1}+\underbrace{\hat{A}_{20} \hat{G}_{-19}}_{\rightarrow 0}+\underbrace{\hat{A}_{40} \hat{G}_{-39}}_{\rightarrow 0}+\ldots
$$


In Ewert et al. ${ }^{5}$ is shown, that the level of the radial filter kernel decays rapidly with growing mode order. In this case, the contribution of filter kernel $\hat{G}_{20}, \hat{G}_{40}, \ldots$ is considerably small (compare with Fig. 4 that shows contour plots of the filter kernel $\hat{G}_{m}\left(\frac{r}{l_{s}^{*}}, \frac{r^{\prime}}{l_{s}^{*}}\right)$ for modes $m=0, m=22$ and $\left.m=44\right)$. That means, for this kind of nozzle trailing edge geometries, in a first attempt it might be sufficient to consider only the 0th azimuthal mode of source variance. In the following sections, CAA computations with only the first six or

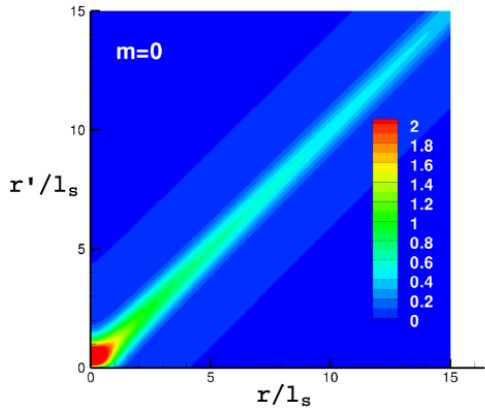

(a) $m=0$

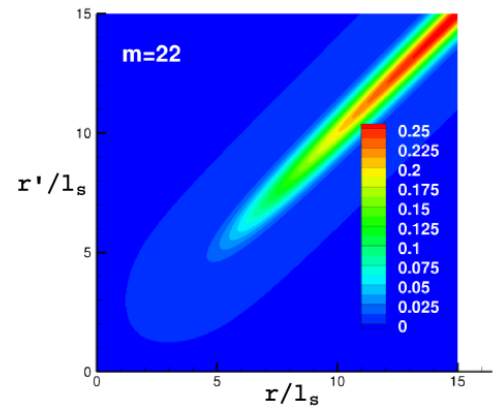

(b) $m=22$

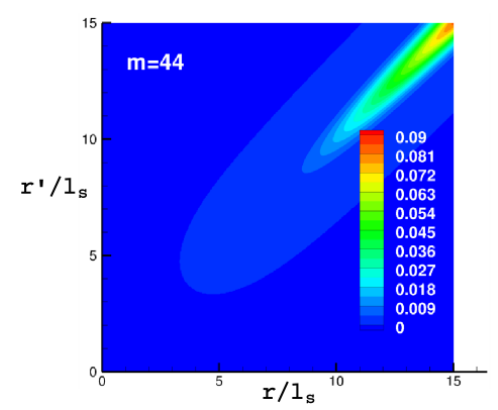

(c) $m=44$

Figure 4. Radial filter kernel $\hat{G}_{0}, \hat{G}_{22}$ and $\hat{G}_{44}$ for a nozzle with 44 serrations

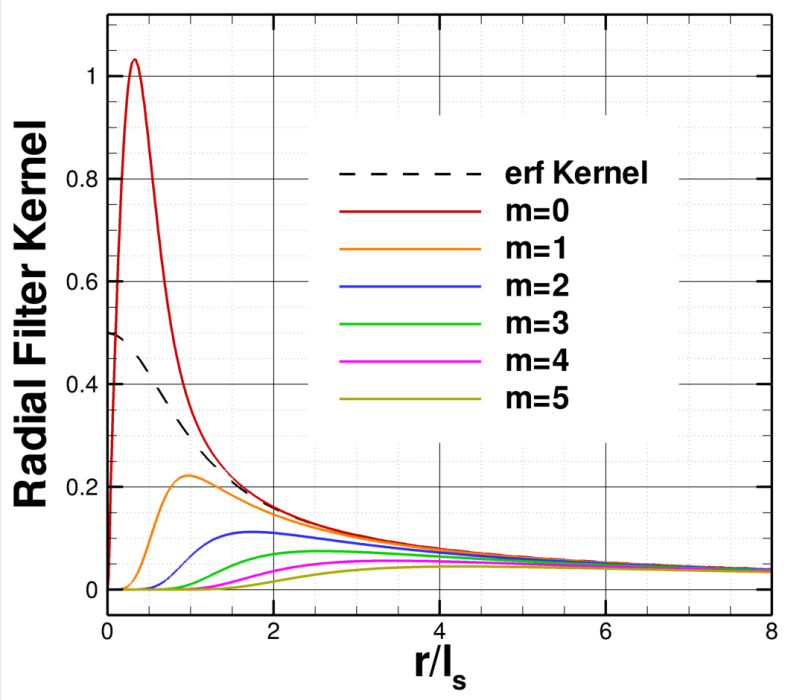

Figure 5. Radial filter kernel for the first 6 azimuthal modes, along the line $\frac{r}{l_{s}^{*}}=\frac{r^{\prime}}{l_{s}^{*}}$

rather ten/eleven azimuthal modes are simulated. The decaying character of the first six modes can be seen in Fig. 5. They strongly differ in a range of $\frac{r}{l_{s}^{*}} \approx 0 \ldots 6$, but coincide for bigger $\frac{r}{l_{s}^{*}}$ values.

\section{Computational Setup}

\section{A. CFD settings}

Previously computed RANS data of cold single stream jet, which was already used for jet noise predictions published in Neifeld et al. ${ }^{6}$ is applied in the present work, too. These RANS calculations with different subsonic Mach numbers $(M a=0.3,0.5,0.6,0.7,0.75,0.8,0.9,0.95)$ in cold condition were calculated for Reynolds numbers ranging between $1.0 \cdot 10^{6}$ to $20 \cdot 10^{6}$. Several jet diameters $(D=0.05 m-1.0 m)$ were computed with a double convergent nozzle geometry. An azimuthal segment of $\phi=30^{\circ}$ is resolved by the 
computational domain. The extension into free-field are 50 jet diameters in downstream direction and 15 jet diameters in radial direction, respectively. For all these configurations a structured grid with $1.5 \cdot 10^{6}$ points was employed. The ambient temperature and density were taken from standard atmosphere as $T_{\infty}=288.15$ $\mathrm{K}$ and $\rho_{\infty}=1.225 \frac{\mathrm{kg}}{\mathrm{m}^{3}}$ in static condition. For all these computations a standard Menter-SST turbulence model was used. Each computation was conducted within 48 hours wall clock time in parallel mode on $32-96$ CPUs.

The RANS solutions of dual stream jets with and without nozzle guide elements (NGE) were computed by Rolls-Royce Deutschland. The ambient Mach number is $M a=0.25$ and the nozzle exit Mach numbers are $M a_{i}=0.75$ and $M a_{o}=0.9$ with an outer nozzle diameter of $D_{j}=0.22 \mathrm{~m}$. The RSM (Reynolds stress model) turbulence model is applied in these computations, which had better agreement with experiments for this configurations than the Menter SST turbulence model.

\section{B. CAA settings}

The CAA computation for jet noise in 3-D modal mode needs an azimuthal mode number adjusted grid. We solve all azimuthal modes simultaneously during one computation. Therefore, for each mode order one $x-r$-plane is needed. Practically, we realize a 3-D grid with the first two coordinate directions resolving the $x-r$-plane whilst the number of $z$-planes defines the mode order resolved (i.e. the $k$-index of the third coordinate direction is directly related to the azimuthal mode order). The CAA computation running on this grid is numerically 2-D. The LEE solved on each $k$-plane is related to the corresponding mode order. In addition we realize complex-valued quantities by introducing two planes with real-valued quantities for each mode order, representing the real and imaginary part, respectively. The results, shown in Sec. V., are computed on such a grid with $12 x-r$-planes, where each plane represents one azimuthal mode. Thus, we are capable of resolving with this mesh 6 azimuthal modes. To resolve frequencies up to Strouhal number of 10 (with $M a=0.3$ ), there was a need for $4.0 \cdot 10^{6}$ points. The extension of CAA domain is 15 jet diameter in radial direction and 30 jet diameter in downstream direction. The RPM source domain encloses a region of $R / D=0.1 \ldots 2$ and $X / D=0 \ldots 30$. The source domain contains 50 streamlines prescribing the moving traces for RPM particles. The number of particles on each streamline is of a dimension of $10^{3}$. The exact number of particles depends on the convective velocity on a streamline, which is prescribed by RANS solution. Each computation was conducted in a time range of $48-168$ hours clock time depending on targeted sample time. For these computations 32 CPUs in parallel mode were used. With above named parameters, it was possible to simulate jet noise samples up to $2.34 \mathrm{~s}$ real time.

\section{3-D modal jet noise results}

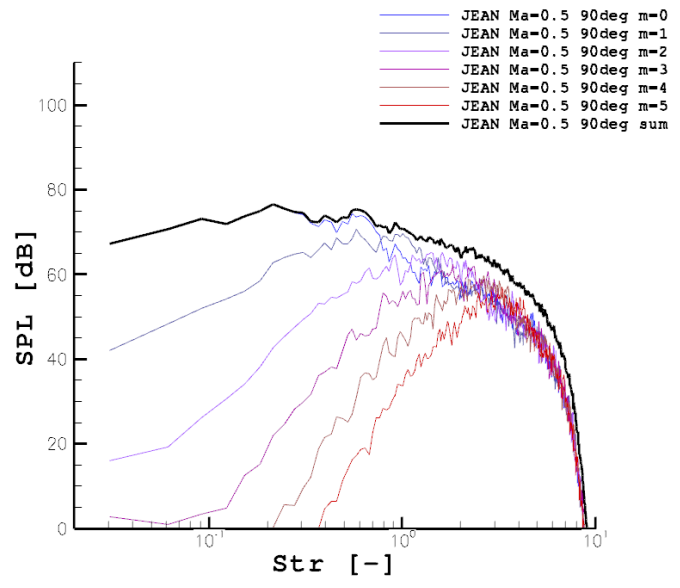

(a) $M a=0.5, \theta=90^{\circ}$

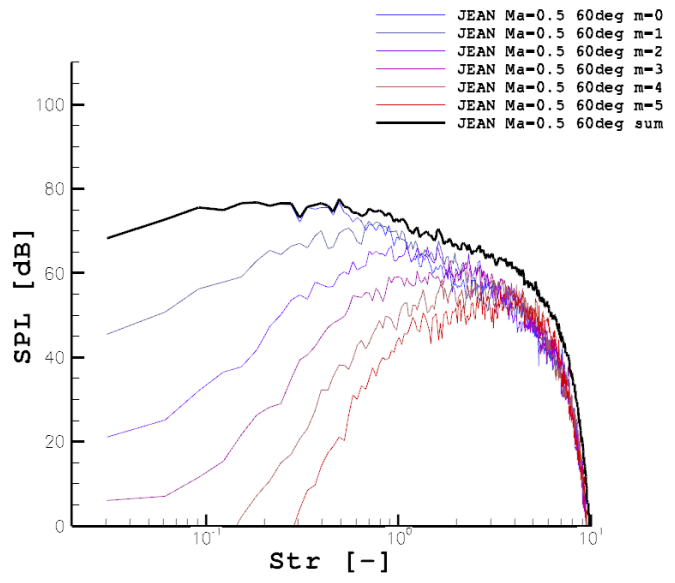

(b) $M a=0.5, \theta=60^{\circ}$

Figure 6. The six considered azimuthal modes spectra and their sum for a cold single stream jet at two different polar angles 


\section{A. Axisymmetric cold single stream jet computations}

To verify the implemented jet noise model of Tam \& Auriault (T\&A) in PIANO, four different RANS solutions with varying jet exit Mach numbers are applied in the CAA computations. The Mach numbers $M a=0.3,0.5,0.7$ and 0.9 are used to verify the Mach scaling law of jet noise. The computed sound pressure levels are expected to deliver the same values as predicted by T\&A model, since the same empirical constants, proposed by the model are also used in PIANO. As reference for the verification, the measurements and the genuine model of a single stream jet noise published in Tam et al. ${ }^{1}$ are taken into account.

In Fig. 6 the spectra of azimuthal modes $m=0, \ldots, 5$ for $M a=0.5$ at observer position $\theta=90^{\circ}$ and $\theta=60^{\circ}$ are depicted. The spectra of remaining Mach numbers are shown in Fig. 17. The distance of sampling positions to the jet axis is $R / D_{j} \approx 10$, which is still in the near-field of a jet. A simplified extrapolation under static condition was conducted to estimate the SPL-values at $R / D_{j}=100$. To obtain the physically relevant prediction, the modal spectra have to be summed up to one spectrum, which are as well shown in the figures. It is noticeable, that the contribution of azimuthal modes to the sum-spectra have always the same pattern. This pattern reveals, that all of the simulated azimuthal modes are important for the shape of the sum-spectra. Each of these single spectra has a frequency range, where the SPL of this azimuthal mode is higher than all the other modes. In other words, the roll-off shape of the sum-spectra at high frequencies is strongly dependent on how many azimuthal modes are considered in the computation. Starting with the zeroth mode, which is responsible for the most contribution at low frequencies, the sum-spectra is build up from succesive azimuthal modes.

A quantitative comparison of resulting sum-spectra with reference spectra is depicted in Fig. 7. As a fine-scale jet noise model the T\&A model should roughly apply to a polar angle range between $\theta=120^{\circ}$ and $\theta=60^{\circ}$. These bounds are shown in Fig. 18. Due to the good match of computed spectra to the model and measurements, we can also state, that the Mach scaling exponent is correctly predicted. These comparisons let us conclude, that the model is properly implemented.

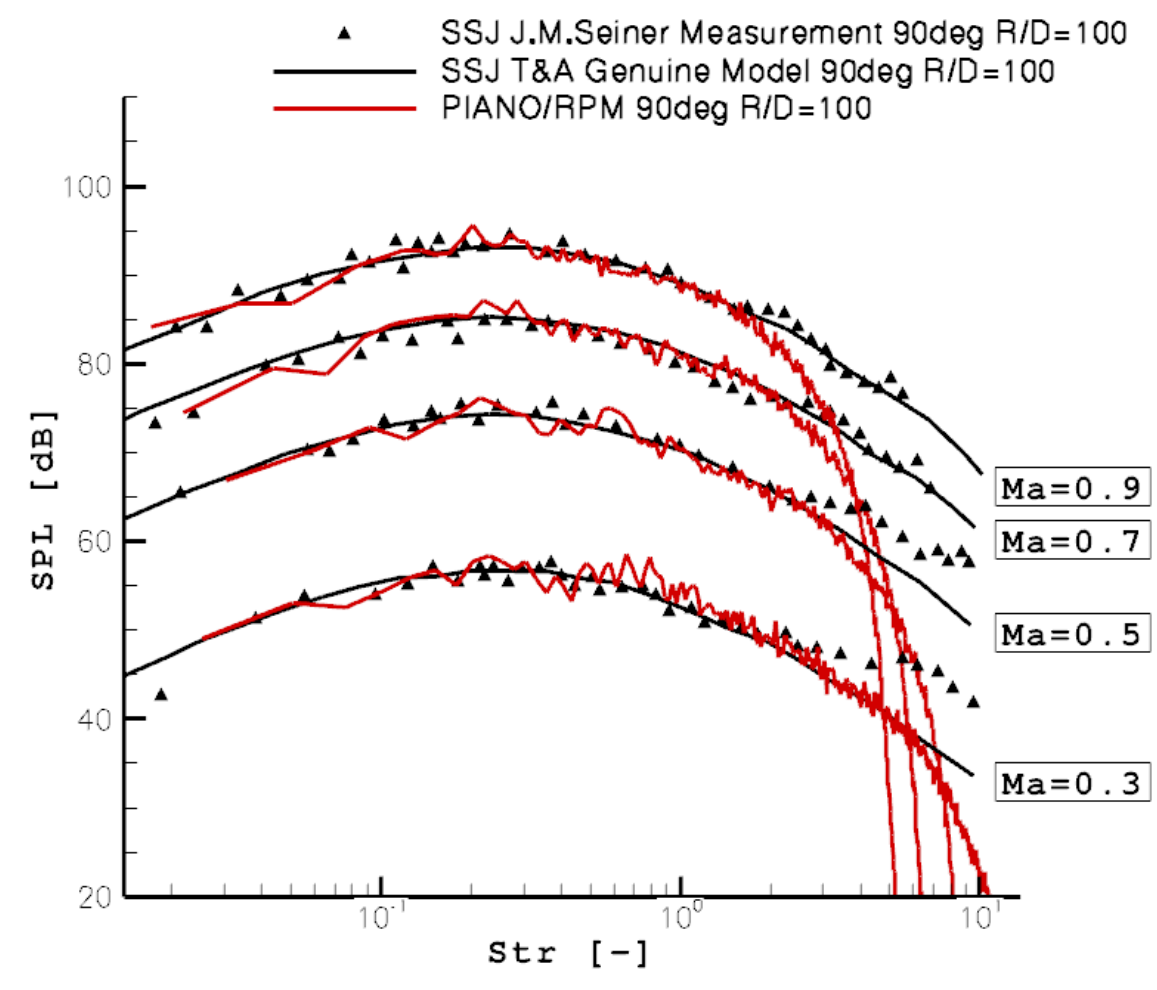

Figure 7. Cold single stream jet spectra obtained by PIANO/RPM at the polar angle $\theta=90^{\circ}$ with Mach numbers $M a=0.3,0.5,0.7,0.9$ in comparison to T\&A model and measurements

If these results are compared to the experimental investigations of Fuchs and Michel, ${ }^{8}$ Juvé et al. ${ }^{9}$ and Brown and Bridges. ${ }^{10}$ the individual contribution of azimuthal modes in experiments has a comparable 
pattern as it is observed in our computations. It is difficult to make any statement at high Strouhal numbers, where the higher azimuthal modes come into account in the computation, since the measurements are evaluated below $S t=0.3$.

\section{B. Influence of azimuthal mean-flow refraction}

To study the effect of azimuthal varying mean-flow on sound refraction in the shear layer, two test computations were conducted. The mean-flow used here is describing a nozzle with NGE, which is nonuniform in azimuthal direction at the nozzle trailing edge. The interest is focused on the refraction effect of sound propagation and if it is sufficient to use only the zeroth azimuthal mode of the mean-flow. The configuration of these computations is in principle the same with the difference, that the mean-flow of the first computation rests on the 3-D RANS data, whereas the second computation uses only the zeroth azimuthal mode of the same RANS solution. The acoustical source in both computations is one periodic monopole positioned at $X / D_{\text {jet }}=0.1$ (or at $X / D_{\text {jet }}=0.2$ ) in the shear layer of the jet (Fig. 8(a)). The difference between the both sound pressure fields then is expressing the error made by considering refraction effects only for the azimuthally averaged mean-flow. The difference between both mean-flows is depicted in Fig. 8(b). Due to this contour plot, there are clearly noticeable some deviations at the nozzle trailing edge. However, the aim is to predict jet noise, emitted in the range of the T\&A model. If we evaluate the pressure samples at the same position as it was done in previous section, i.e. at $R / D \approx 10$ and in polar angle range of $\theta=60^{\circ} \ldots 120^{\circ}$, the difference is considerably small. Two extracts of pressure samples at $\theta=90^{\circ}$ are depicted in Fig. 8(c,d). The difference of both signals is of order $\Delta L_{p}=0.1 \ldots 0.3 \mathrm{~dB}$, in dependence on polar angle. This would imply that the error caused by neglecting of higher azimuthal mean-flow modes is relatively small for this kind of nozzle trailing edge.

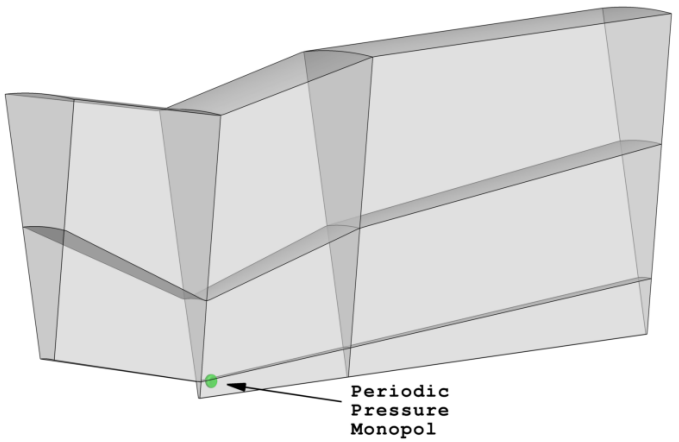

(a) Monopole position

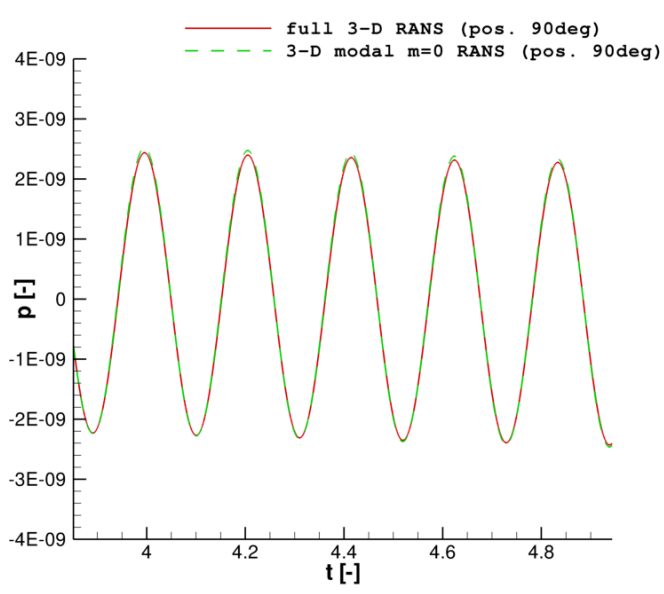

(c) Pressure signals at $R / D_{j e t}=10$ for a monopole positioned at $X / D_{\text {jet }}=0.1$

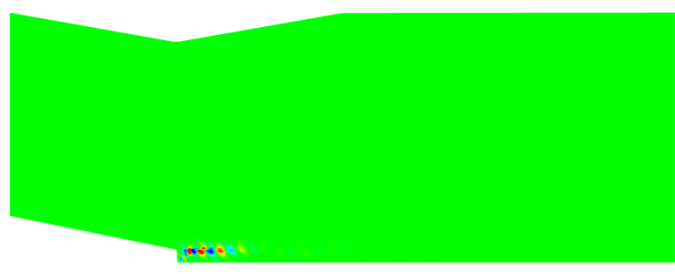

(b) Pressure difference

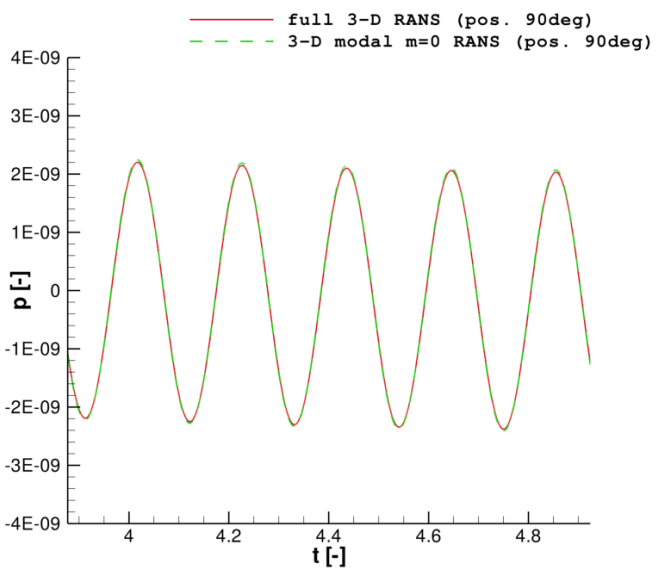

(d) Pressure signals at $R / D_{\text {jet }}=10$ for

a monopole positioned at $X / D_{\text {jet }}=0.2$

Figure 8. Refraction effect of serrations studied via periodic pressure monopole 


\section{Computation of a nozzle with azimuthal varying mean-flow}

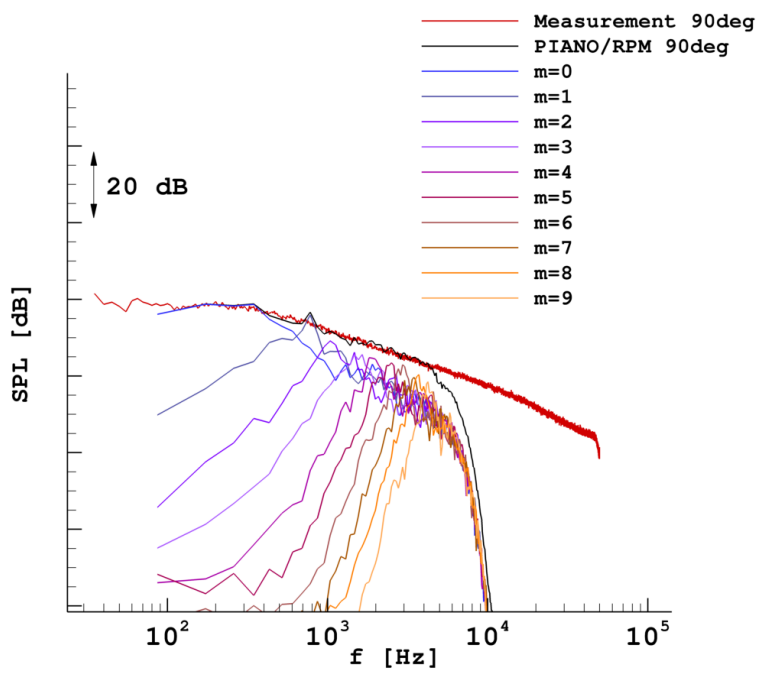

(a)

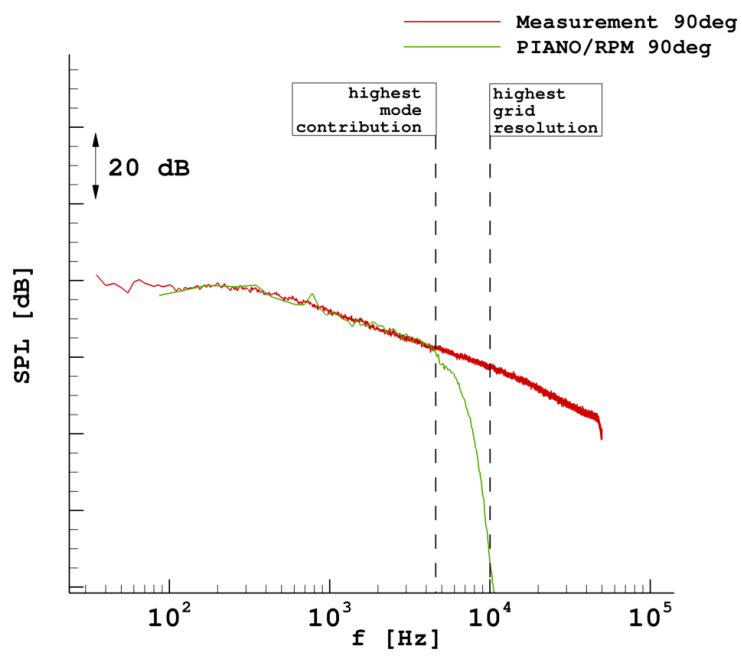

(b)

Figure 9. Cold dual stream jet (with nozzle guide elements) spectrum obtained by PIANO/RPM at the polar angle $\theta=90^{\circ}$ in comparison to measurement; (a) Spectra of each azimuthal mode and its sum-spectrum, (b) highest mode contribution and grid resolution of the sum-spectrum

In this section, the CAA computations of dual-stream jet configurations are considered. The focus is on the prediction of the acoustical benefit of nozzle guide elements (NGE) via the fine-scale noise radiated in the polar angle range between $60^{\circ}$ and $120^{\circ}$. Herein, the major difficulty is, that the application of NGE generates azimuthal variation of the flow, which would lead to a more complex dependency of azimuthal modes (between perturbation and mean-flow quantities) in this approach.

As it was found in previous section with the test case of sound refraction by azimuthal varying meanflow, the assumption to consider only the zeroth azimuthal mean-flow is expected to cause negligibly small deviation to completely considered mean-flow. Also the consideration of higher azimuthal modes of RPMsources (for this kind of NGE the order of azimuthal mode is around 20) can be neglected as it is described in Sec. III.B. That means, if the effects of the azimuthal mode generated by NGE are considerably small, the computation for nozzle configuration with NGE can be processed in the same way as it was done for the single stream jet.

The results of a CAA computation with 10 azimuthal modes for a dual-stream nozzle with NGE are depicted in Fig. 9. As it was found for single stream jet, similar behavior of individual azimuthal mode spectra is found here, too. The sum spectrum is compared to the experimental spectrum, which was measured (QinetiQ) for the same nozzle configuration and can be used as reference spectrum. Both spectra, the experimental and the sum spectrum of CAA computation, match very well over a broad frequency range despite the assumptions mentioned above. The shape of the sum spectrum is comparable to measurements only to a frequency, where the highest considered azimuthal mode contribution ends. That position is marked with the first dashed line in Fig. 9(b). Despite the grid resolution is not reached at that frequency, a sharp drop off of amplitude is observable, since contribution of higher azimuthal modes not considered is missing. Consequently, to get the sum spectrum comparable to measurements up to the grid resolution, several additional azimuthal modes higher than $m=9$ have to be taken into account in the computation.

For the prediction of the acoustical effect of NGEs a second computation of the same configuration without NGE was conducted and compared to the results of Fig. 9. Both CAA spectra are depicted in Fig. 10. As this comparison reveals, with such an approach, it is possible to predict quite small differences in amplitudes between different configurations (in order of $1 d B$ ). Accordingly to $\theta=90^{\circ}$, the agreement to measurement spectra of amplitude difference between the both configurations can be observed for the whole polar angle range between $60^{\circ}$ and $120^{\circ}$. Valuable information to similar investigation at Technical University of Berlin was considered from the results of computations, using a direct method (Detached Eddy Simulation (DES)), which were published by Eschricht et al. ${ }^{17,18}$ 


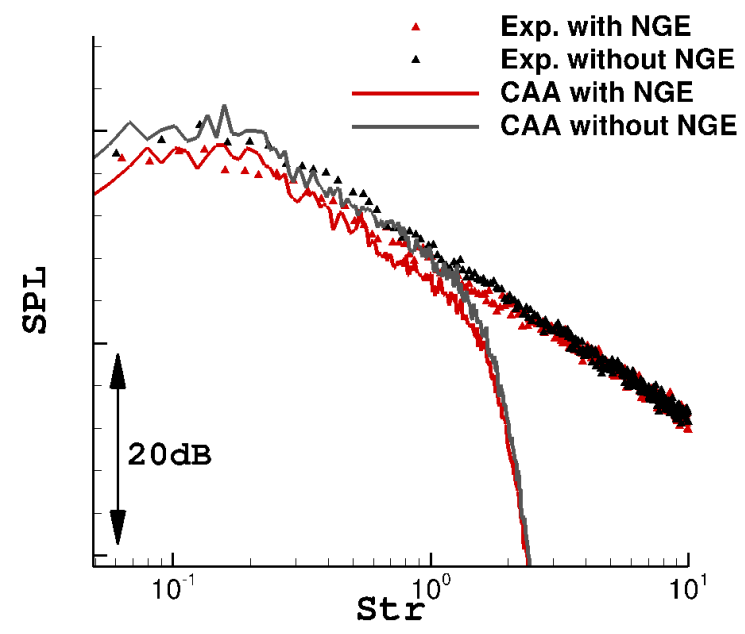

(a)

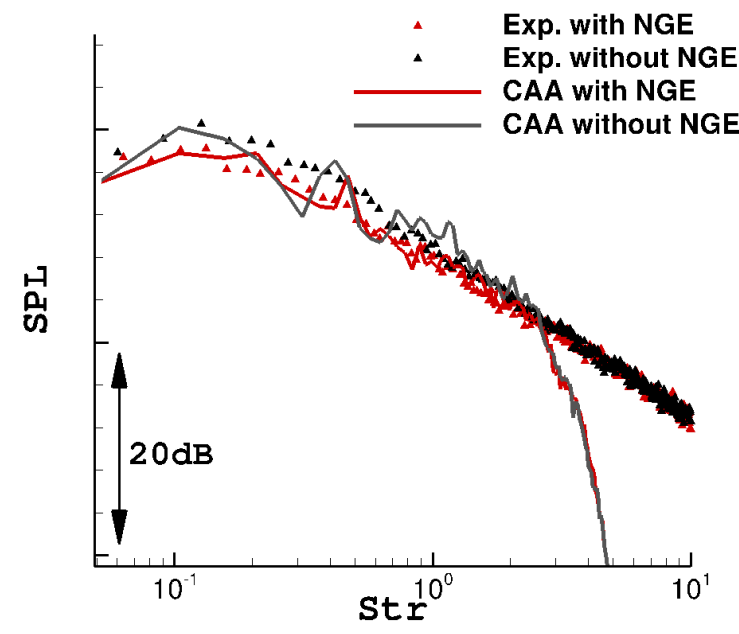

(b)

Figure 10. Cold dual stream jet at the polar angle $\theta=90^{\circ}$, comparison of configurations with and without nozzle guide elements (NGE) with measurements as reference; (a) 6 considered azimuthal modes, (b) 10 considered azimuthal modes on a finer grid

Because of relatively small computational effort needed for this hybrid method, it could take root as an efficient tool for design purposes of nozzle geometries. Numerous configurations with geometry variations affecting sound radiation can be predicted within short terms, since one computation is a matter of some days. With the advantage that for the CAA computations the nozzle geometry is not obviously needed, the bigger effort is to obtain a 3-D RANS solution with the resolved nozzle geometry in it.

\section{Extrapolation into far-field}

\section{A. Method}

To analyze jet spectra in the far-field, the near-field data of the modal CAA/RPM computations is extrapolated with a Ffowcs-Williams \& Hawkings method. An efficient approach to do this in combination with the near-field modal jet noise computations is to use a modal Ffowcs-Williams \& Hawkings (FWH) method, e.g. introduced by Weckmüller et al. ${ }^{15}$ in the frequency domain formulation. We apply a time-domain formulation for our broadband problem. The advantage here is, that instead of to solve a whole source enclosing surface integral, just a line integral has to be solved. Hence, a drastically reduced amount of data has to be stored and processed.

If we take the convenient form of the FWH-integral in 3-D,

$$
4 \pi c_{0} \rho^{\prime}(\mathbf{x}, t)=\frac{\partial^{2}}{\partial x_{i} \partial x_{j}} \int_{V}\left(\frac{T_{i j}}{r\left|1-M a_{0}\right|}\right) \mathrm{d} V-\frac{\partial}{\partial x_{i}} \int_{S}\left(\frac{p^{\prime} \delta_{i j} \cdot n_{j}}{r\left|1-M a_{0}\right|}\right) \mathrm{d} S+\frac{\partial}{\partial t} \int_{S}\left(\frac{\rho_{0} u_{n}^{\prime}}{r\left|1-M a_{0}\right|}\right) \mathrm{d} S,
$$

some simplifications can be assumed, if it is adjusted to the approach. Due to the generation of RPM-sources, there is clearly defined source domain, which spacial extension is known to us. Therefore, it is always possible to define a FWH-surface, which completely enclose all the RPM-sources. So, it is obvious not to consider the term with the Lighthill's stress tensor (quadrupole source term), which simplifies the integral to the following form

$$
4 \pi c_{0} \rho^{\prime}(\mathbf{x}, t)=\frac{\partial}{\partial t} \int_{S}\left(\frac{\rho_{0} u_{n}^{\prime}}{r\left|1-M a_{0}\right|}\right) \mathrm{d} S-\frac{\partial}{\partial x_{i}} \int_{S}\left(\frac{p^{\prime} \delta_{i j} \cdot n_{j}}{r\left|1-M a_{0}\right|}\right) \mathrm{d} S .
$$

For the remaining both surface integrals (loading noise and thickness noise) the near-field distribution of acoustical quantities on a defined surface has to be provided. Since in our case the near-field computations 

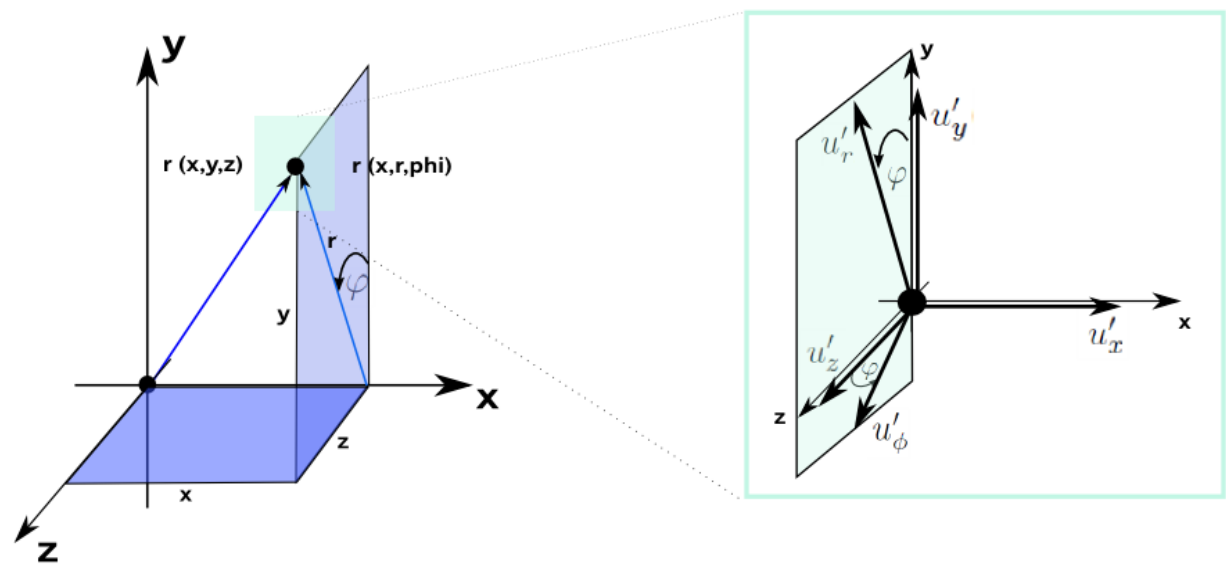

Figure 11. Transformation of perturbation velocities into cartesian coordinate system

are performed in Fourier series decomposition, these quantities have $x, r, t, m$-dependency. To adapt the near-field output to Eq. (35) there are two options. The first one is to reduce the integrals (35) to line integrals with $x, r, t, m$-dependency, where the integration in azimuthal direction is solved analytically prior to the FWH extrapolation of the CAA data, which was done in Weckmüller et al. ${ }^{15}$ The second option is to generate the azimuthal distribution of these values from the modal near-field data and apply the obtained 3-D surface to (35). This option is less efficient, since additional computational time is required for the extension in third dimension and bigger number of discretization elements have to be processed. Nevertheless, at the moment the latter is used for the far-field extrapolation allowing the application of the well established APSIM code of DLR which solves Eq. (35). The time effort for such kind of extrapolation is approximately one day on one CPU for one azimuthal mode. Since the needed simulation time is smaller than that of the simulation step with stochastic sound sources, the higher simulation time of a 3-D FWH procedure is acceptable.

The sketch, depicted in Fig. 12, is illustrating the principle of FWH-geometry definition, where the values for the extrapolation are sampled. In principle, instead of a surface, which should enclose the source region, a line is used to represent the three-dimensional cylindrical geometry. To obtain the full surface from the lines (considering velocity transformation in Fig. 11) following equations are applied:

$$
\begin{gathered}
p^{\prime}(x, r, \phi, t)=p_{\Re}^{\prime}(x, r, t) \cdot \cos (m \phi) \\
\rho^{\prime}(x, r, \phi, t)=\rho_{\Re}^{\prime}(x, r, t) \cdot \cos (m \phi) \\
u_{x}^{\prime}(x, r, \phi, t)=u_{x, \Re}^{\prime}(x, r, t) \cdot \cos (m \phi) \\
u_{y}^{\prime}(x, r, \phi, t)=\left[u_{r, \Re}^{\prime}(x, r, t) \cdot \cos (\phi)+u_{\phi, \Re}^{\prime}(x, r, t) \cdot \sin (\phi)\right] \cdot \cos (m \phi) \\
u_{z}^{\prime}(x, r, \phi, t)=\left[-u_{r, \Re}^{\prime}(x, r, t) \cdot \sin (\phi)+u_{\phi, \Re}^{\prime}(x, r, t) \cdot \cos (\phi)\right] \cdot \cos (m \phi)
\end{gathered}
$$

For symmetry reasons the imaginary part of the complex-valued quantities provided by the stochastic simulation does not contribute to the far-field values if an observer position at $\phi=0^{\circ}$ azimuthal angle is chosen. This in general does not pose limitations on the applicability of the method. As it can be seen in Fig. 12, there are two types of lines - the main FWH-line and the sidelines. The results of the extrapolation shown in the next section are computed using only the main line. It is expected, that the sidelines are less important for the regarded polar angle range, if the FWH-surface is close enough to the jet axis.

To get comparable results to the measured spectra, each of considered azimuthal mode has to be extrapolated and summed up to a single spectrum. Since the azimuthal modes of the extrapolation are not coupled, it is possible to perform these computations in parallel or sequentially. 


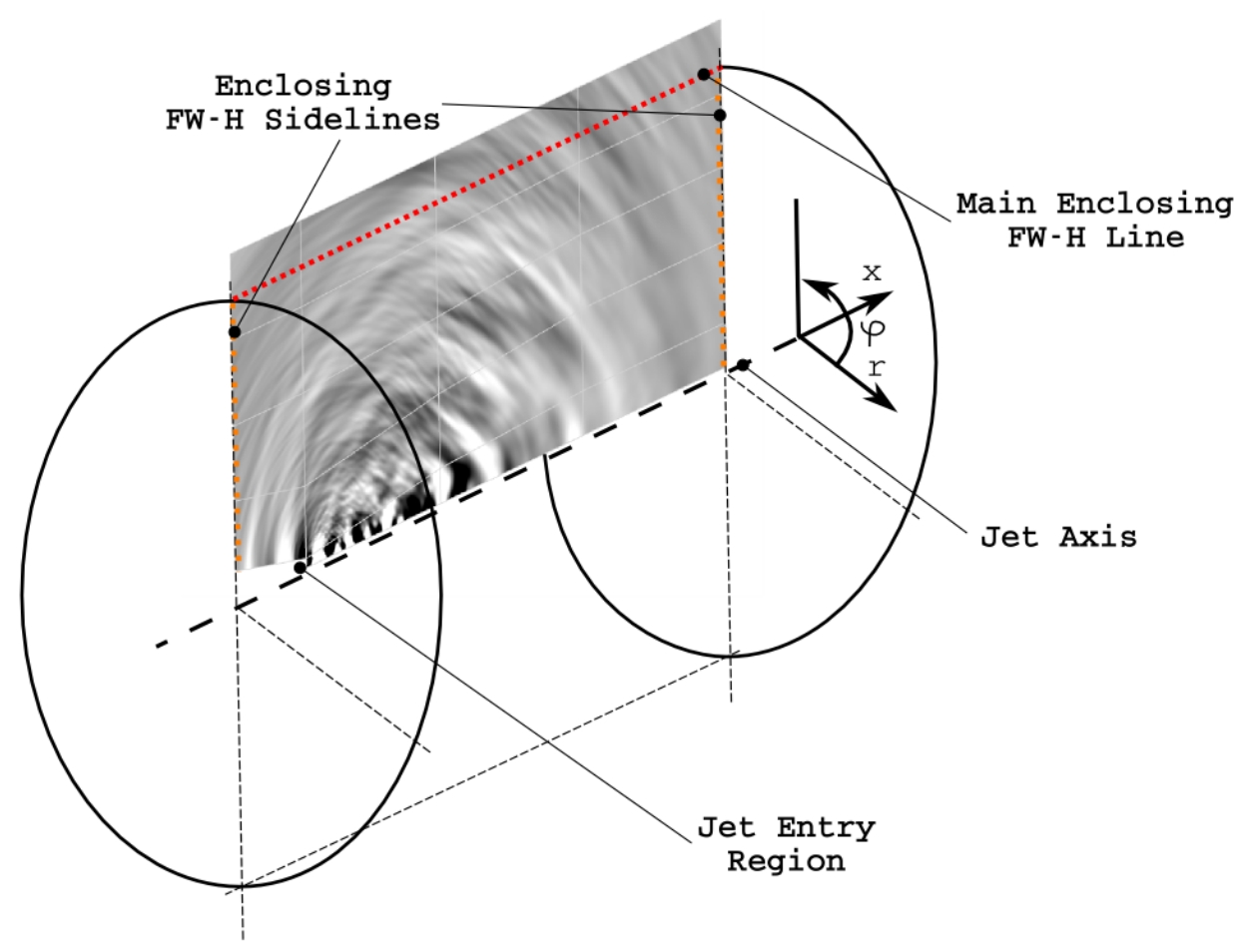

Figure 12. Ffowcs-Williams \& Hawkings enclosing geometry definition

\section{B. Results of the extrapolation}

The extrapolation is applied to a computational case of dual stream nozzle with plain nozzle trailing edge in forward flight, where the precomputed CAA solution of a 3-D modal computation is used as input data. Three different FWH-surfaces with different radial distances to the jet axis, namely $R_{1} \approx 2.5 \cdot D_{j}, R_{2} \approx 5.0 \cdot D_{j}$ and $R_{3} \approx 10.0 \cdot D_{j}$ were defined prior to the CAA computation and are used for the analysis.

The extrapolation results for the surfaces $R_{1}$ and $R_{2}$ at polar angle $\theta=90^{\circ}$ are depicted in Figs. 13 . and 14. The surface $R_{3}$ was not further considered. The spectra for the polar angles $\theta=60^{\circ}$ and $\theta=120^{\circ}$, which are the bounds of T\&A model are shown in Figs. 21. and 23. In Figs. 20. and 22. the pressure distribution on the FWH-surface is depicted for the first six azimuthal modes related to the surface positions $R_{1}$ and $R_{2}$.

To get a reference spectra for the extrapolation, a position at $\theta=90^{\circ}$ was chosen in the CAA domain, which is labeled in the figures as verification position. The observer position for FWH-computed spectra was chosen at the same position. The comparison of both spectra reveals (refer to Fig. 13(b) and 14(b)) a good agreement for surface data positions $R_{1}$ and $R_{2}$. It is noticeable at this point, that the $R_{1}$-spectrum is considerably faster computed than the $R_{2}$-spectrum. Since the surface element width of both surfaces is the same, the number of elements of $R_{2}$ is correspondingly higher due to the bigger radius. On the other hand the frequency resolution of $R_{1}$ is higher than that of $R_{2}$. The reason for that is, that the CAA grid resolution in radial direction becomes slightly coarser letting the higher frequencies dissipate on the way to the reference position. Whilst the distance bypassed with the extrapolation is without any loss in frequency resolution.

Judging from these results, it is not necessary to start with the extrapolation at $R_{2}$ and it is more efficient to use the position $R_{1}$. Despite the nozzle upstream region of $R_{1}$ is not covered by the main FWH-line, the spectra in the regarded polar angle range have good agreement to those of CAA. Also the FWH-sidelines were not included for these extrapolations, which means that these lines can be neglected.

The investigation of jet noise extrapolation of Shur et al. ${ }^{16}$ shows similar FWH-surface definition variation, whereas there they start with the biggest radius that is comparable to $R_{1}$. The other two surfaces are even closer to the source region of the jet, for which the consideration of the FWH-sidelines is not negligible 


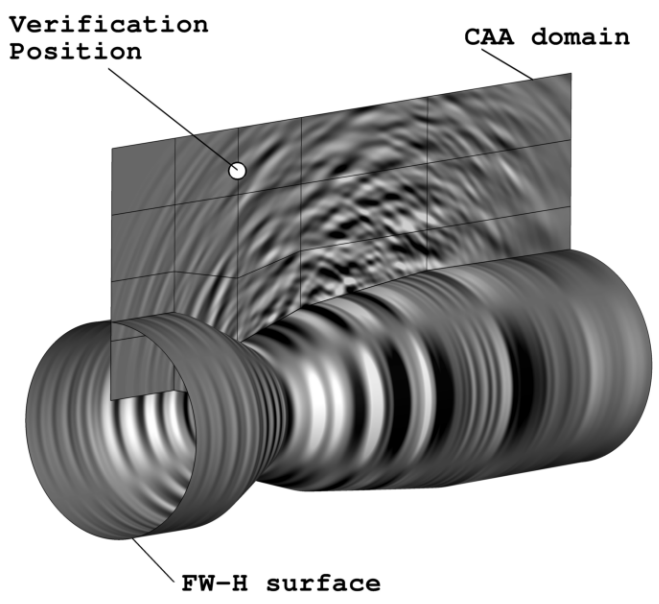

(a) Sound pressure on FWH-surface and CAA domain for $m=2$

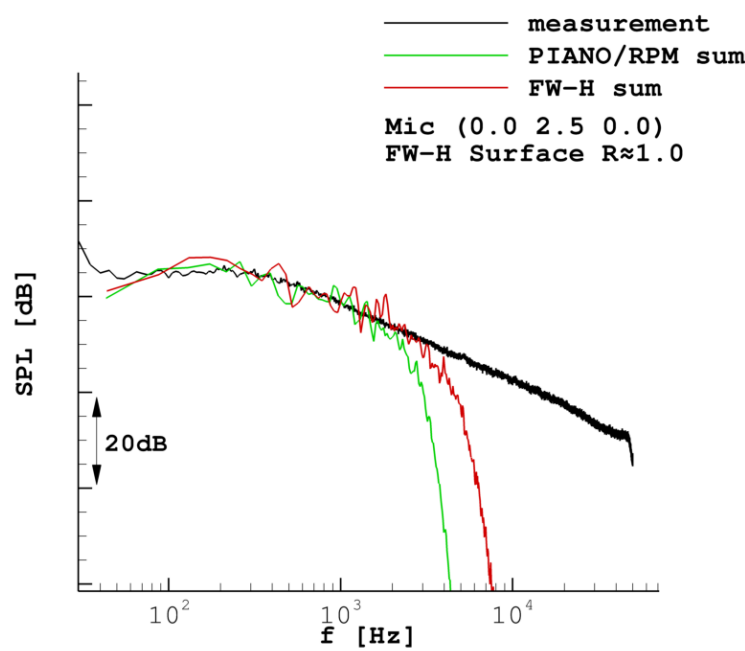

(b) Sum spectra of CAA computation and FWH extrapolation at the same observer position

Figure 13. Test case for the modal FWH extrapolation with surface at $R \approx 5.0 \cdot D_{j}$

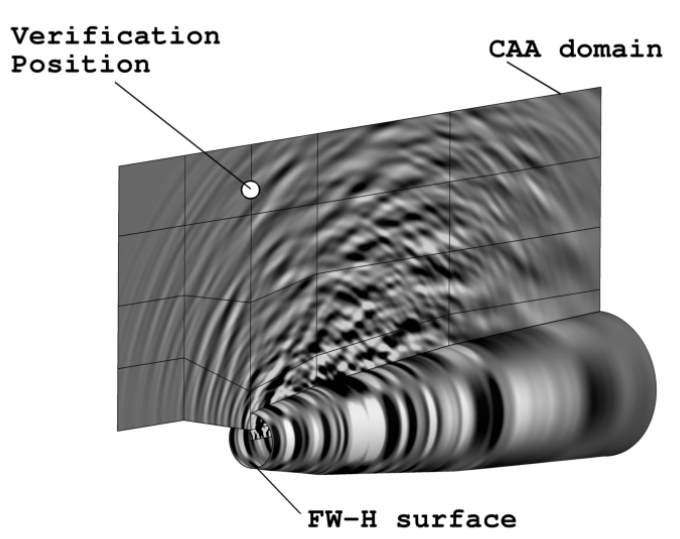

(a) Sound pressure on FWH-surface and CAA domain for $m=2$

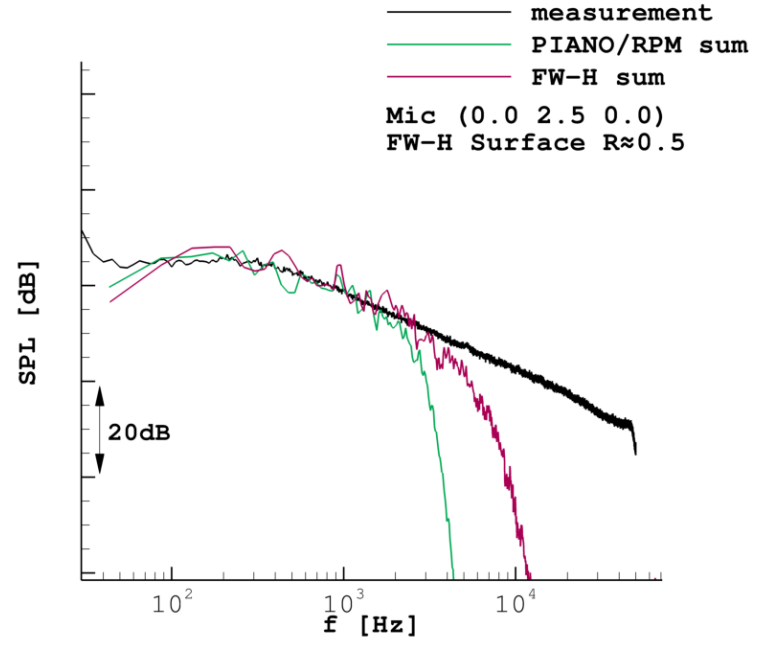

(b) Sum spectra of CAA computation and FWH extrapolation at the same observer position

Figure 14. Test case for the modal FWH extrapolation with surface at $R \approx 2.5 \cdot D_{j}$

as their results reveal. Taking this in account, the $R_{1} \approx 2.5 \cdot D_{j}$ seems to be a favorable radial distance for the extrapolation.

\section{Optimized computations}

With the FWH results shown in previous section, some improvements of the CAA computation are possible in combination with the extrapolation method. As it can be seen in the results of $R_{1}$ and as well in the FWH investigation of Shur et al., ${ }^{16}$ this distance is sufficient to predict accurately the far-field spectra. Therefore, a large number of CAA grid points can be dropped without any loss of spectral quality, if the CAA domain outside of FWH-surface is not of any interest.

Considering the position of the FWH-surface, the CAA domain was reduced to a radial extension of $3 D_{j}$ without changing the axial extension. Hence, more than a half of CAA grid points were saved by reducing 


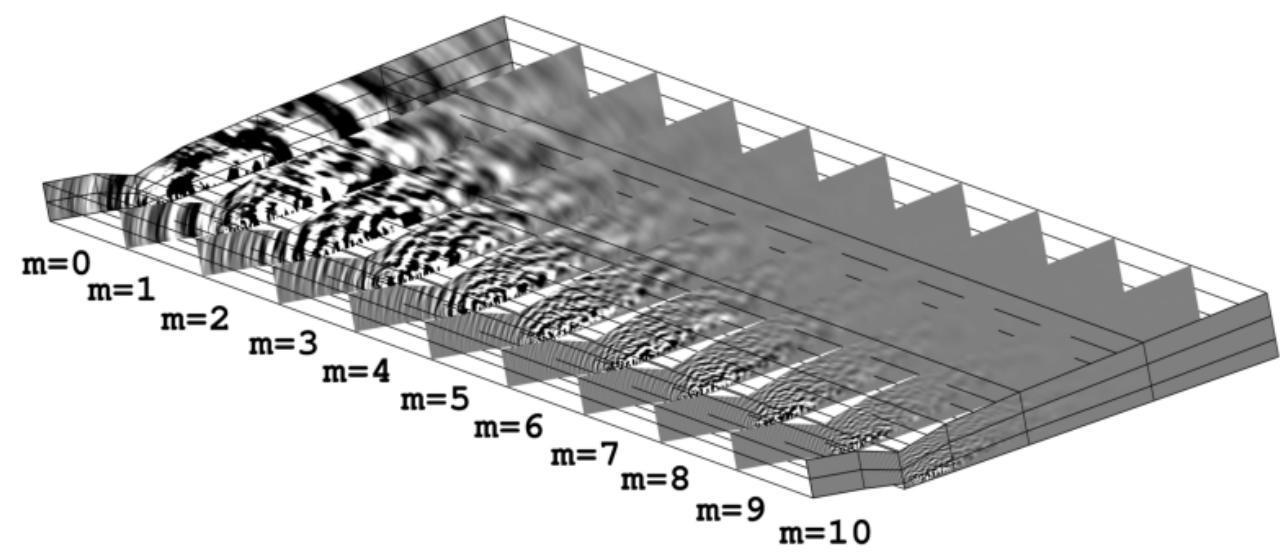

Figure 15. Sound pressure contour plots of the 11 considered azimuthal modes (cold SSJ, $\left.M a=0.9, D_{j}=0.5 m\right)$

the computational domain, which were spent in addition on the smaller simulation domain to increase the frequency resolution. For this computation 11 azimuthal modes are considered (refer to Fig. 15.). This enable us to increase drastically the frequency resolution, whilst the computational effort increases roughly by one day, due to the computational effort of the extrapolation. The gain of these improvements is for a SSJ with $M a=0.9$ a shift of highest resolution from $S t \approx 5.0$ to $S t \approx 20.0$ as shown in Fig. 16 .

For this case, the CAA spectrum in the near-field has been evaluated at polar angle $\theta=90^{\circ}$ with $R / D_{j}=2.5$ distance to the jet axis. This sum spectrum is shown in Fig. 16(a) as a comparison to the previously computed spectrum for the same jet. The observer position of non-optimized PIANO/RPM spectrum has been evaluated as well in the near-field, but with considerably larger distance to the jet axis, namely at $R / D_{j}=10.0$. Both spectra have been extrapolated with simple scaling of sound pressure level with the inverse of radial distance. In the low Strouhal number range, the simply extrapolated optimized computational spectrum deviates from the targeted far-field spectrum shape. Apparently, the optimized near-field spectrum is too close to the jet axis to predict the characteristic spectrum shape already in its asymptotic far-field limit. To verify that the weaker amplitudes at low Strouhal numbers are effects of the near-field position, the same comparison was conducted with the modal FWH extrapolated spectrum in the far-field using the optimized computation. This comparison is shown in Fig. 16(b). There, it readily can be seen, that both spectra coincide over the whole Strouhal number range with the difference, that the optimized computation spectrum is reaching up to $S t=20.0$. From this we can deduce, that for a computational domain as it was realized in the optimized computation, the modal FWH extrapolation is unavoidable, whilst in the outer jet noise near-field (around $R / D_{j}=10.0$ ) the typical shape of the fine-scale G-Spectrum is already present.

\section{Summary and Conclusions}

The application of an efficient approach for jet noise computations of isolated axisymmetric and quasi axisymmetric nozzle configurations, based on Fourier series decomposition is described in this paper. The sound generation is realized with the random particle method (RPM) using the source model of Tam \& Auriault for cold fine-scale jet noise. The genuine T\&A model is solved in the frequency domain, therefore it needs individual (adjoint) CAA compuations for each observer position and frequency band. A broadband stochastic method solved in the time-domain for each azimuthal mode order needs only about six axisymmetric, i.e. quasi 2-D computations to reach a jet Strouhal number of $S t=10$. Furthermore, the latter apporach can be applied to spreading (i.e. non-parallel) jets without increased computational effort The sound propagation is performed with the linearized Euler equations (LEE). The extrapolation into far-field is analyzed by the Ffowcs-Williams \& Hawkings (FWH) method. The whole approach is based on azimuthal mode decomposition, which enables to switch the problem from one 3-D computation to a few axisymmetric 2-D computations. This transformation of the problem results in a drastic increase of efficiency whilst maintaining qualitative good results. The methodology, containing some aspects of empiricism and modeling, 


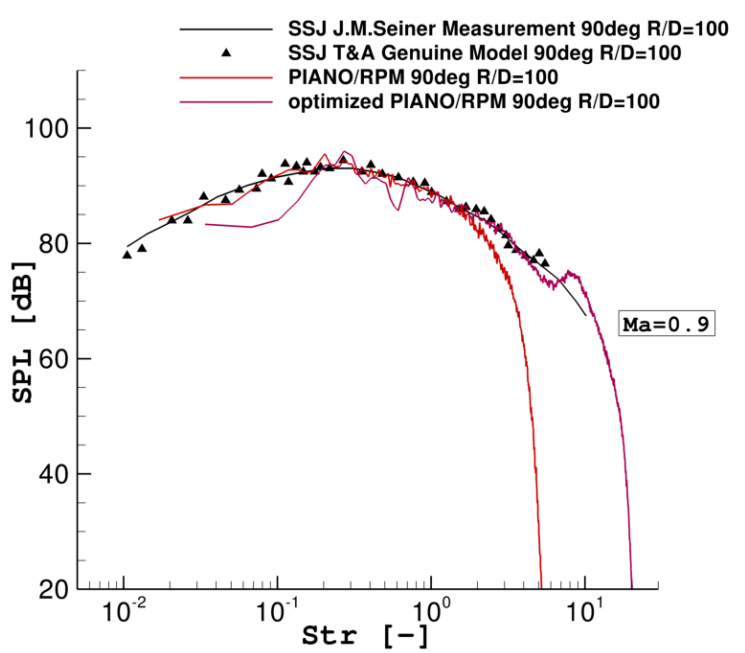

(a) Simplified extrapolation of near-field spectrum computed in the CAA domain at $R / D_{j}=2.5$

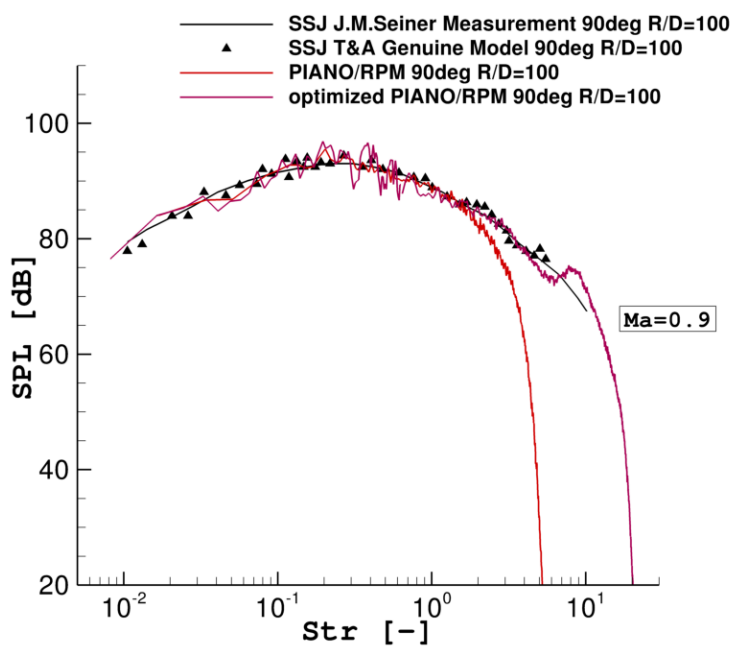

(b) Far-field spectrum computed with the modal FWH extrapolation

Figure 16. Spectra of optimized CAA computation in comparison to prior spectra

can be deemed a semi-empirical jet noise model solved with state-of-the-art CAA methods, which can be regarded as complementary to direct methods.

With the shown computational results in previous sections, we can state that this approach is verified as well as validated. The configurations of a cold single stream jet with different Mach numbers were used for the verification and validation, which were evaluated in the polar angle range of $\theta=60 \ldots 120^{\circ}$ starting from the jet axis. The comparison of these computations to original results (published as measured and modeled spectra) of Tam \& Auriault performs considerably good match. Furthermore, noise predictions in the same polar angle range were conducted for more complex nozzle geometries i.e. dual-stream nozzles with and without nozzle guide elements. The results of these computations show that this approach is capable to predict the difference in amplitudes generated by different nozzle geometries, i.e. the application of NGEs, which leads to a reduction of fine-scale noise in order of $1-2 d B$ relative to a nozzle without any nozzle lip treatment can be predicted.

The combination of CAA near-field computation with the FWH extrapolation makes it possible to neglect a large part of the CAA computational domain. With this, reasonably higher resolution of spectra can be achieved $(S t=20)$, since the grid points saved can be reused in the resolved domain, whilst keeping the computational time the same. However, the near-field spectra at this radial distance is not representative anymore to the far-field spectra, which makes the FWH extrapolation step mandatory.

Practically, a spectral resolution of Strouhal numbers up to $\mathrm{St}=15$ is deemed sufficient for jet engine nozzle design. From our findings, it can be deduced that approximately 8 azimuthal modes are necessary to resolve this Strouhal number. This in principle would allow for further savings in grid points compared to the $\mathrm{St}=20$ simulation presented in the paper, with an according reduction in the simulation time. We have simulated and analyzed noise spectra for nozzle with and without nozzle lip treatments. Comparison with experimental data shows very good agreement in the predicted noise reductions obtained for the nozzle with lip treatment in comparison to the untreated reference configuration, which are of the order of $2 d B$. For higher Strouhal numbers both experimentally measured nozzle spectra converge, i.e. the noise reduction benefit vanishes. A similar trend is found in the simulation, i.e. a convergence of both spectra in the same Strouhal number range. Thus, for the two nozzle configurations, our numerical results are successfully validated with the measurements.

To conclude, the source model of jet mixing noise is proposed for a single stream jet. But, it is also applicable to diverse more complex nozzle configurations, since the basic mechanism of sound generation in the shear layer remains the same with different effects of sound refraction and changing statistical correlations. Therefore, predictions for a wide range of complex nozzle configurations can be conducted with this kind of computations with relatively small effort, which might be interesting for nozzle design purposes. 


\section{Acknowledgments}

This work has been conducted in the framework of the OPTITHECK project in collaboration with RollsRoyce Deutschland. Special thanks to Drs. M. Rose and M. Trumper for their valuable contribution and for providing CFD and experimental data. Furthermore, thanks to Prof. J.W. Delfs of the departement of Technical Acoustics for his guidance during the project. The computational time is provided by Center for Computer Applications in AeroSpace Science and Engineering $\left(\mathrm{C}^{2} \mathrm{~A}^{2} \mathrm{~S}^{2} \mathrm{E}\right)$.

\section{References}

${ }^{1}$ Tam, C.K.W. and Auriault, L., Jet mixing noise from fine-scale turbulence, AIAA Vol. 37, No. 2, February 1999.

${ }^{2}$ Pope, S., Turbulent Flows, Cambridge University Press, 2000.

${ }^{3}$ Ewert, R., RPM - the fast Random Particle-Mesh method to realize unsteady turbulent sound sources and velocity fields for CAA applications, AIAA 2007-3506.

${ }^{4}$ Ewert, R., Dierke, J., Siebert, J, Neifeld, A., Appel, C., Siefert, M. and Kornow, O., CAA Broadband Noise Prediction for Aeroacoustic Design, Journal of Sound and Vibration, Volume 330, Issue 17, p. 4139-4160.

${ }^{5}$ Ewert, R., Neifeld, A. and Fritzsch, A., A 3-D modal stochastic jet noise source model, AIAA 2011-2887, 17th AIAA/CEAS Conference.

${ }^{6}$ Neifeld, A. and Ewert, R., Jet Mixing Noise from Single Stream Jets using Stochastic Source Modeling, AIAA 2011-2700, 17th AIAA/CEAS Conference.

${ }^{7}$ Maestrello, L., Statistical Properties of Sound and Source Fields of an Axisymmetric Jet, 4th AIAA Conference, Atlanta, Georgia, Oct. 3-5, 1977

${ }^{8}$ Fuchs, H.V and Michel, U., Experimental Evidence of Turbulent Source Coherence Affecting Jet Noise, AIAA J., Vol. 16, No. 9, Sep. 1978.

${ }^{9}$ Juvé, D., Sunyach, M. and Comte-Bellot, G., Filtered Azimuthal Correlations in the Acoustic Far Field of a Subsonic Jet, AIAA J., Vol. 17, No.1, 1979

${ }^{10}$ Brown, C.A., and Bridges, J., Acoustic Efficiency of Azimuthal Modes in Jet Noise Using Chevron Nozzles, NASA/TM2006-214364

${ }^{11}$ Cavalieri, A.V.G., Jordan, P., Gervais, Y. and Colonius, T., Axisymmetric superdirectivity in subsonic jets, AIAA 20112743, 17th AIAA/CEAS Conference.

${ }^{12}$ Koenig, M., Cavalieri, A.V.G., Jordan, P. and Gervais, Y., Intermittency of the azimuthal components of the sound radiated by subsonic jets, AIAA 2011-2746, 17th AIAA/CEAS Conference.

${ }^{13}$ Cavalieri, A.V.G., Rodriguez, D., Jordan, P., Gervais, Y. and Colonius, T., Inlet Conditions and Wave-Packets in Subsonic Jet Noise, Turbulence and Shear Flow Phenomena, Ottawa, Canada, July 28-31, 2011.

${ }^{14}$ Guérin, S., Impact of Serrations on the Circumferential Structure of Jet Turbulence and Relation to Jet Noise Reduction, AIAA 2008-3025, 14th AIAA/CEAS Conference.

${ }^{15}$ Weckmüller, C., Guérin, S., Wellner, J. and Michel, U., Ffowcs Williams \&s Hawkings Formulation for the Convective Wave Equation and Permeable Data Surface, AIAA 2010-3710, 16th AIAA/CEAS Conference.

${ }^{16}$ Shur, M.L., Spalart, P.R. and Strelets, M.Kh., Noise prediction for increasingly complex jets. Part I: Methods and tests, Int. J. of Aeroacoustics, Vol. 4, No. 3+4, 2005

${ }^{17}$ Eschricht, D., Yan, J., Michel, U. and Thiele, F., Prediction of Jet Noise from a Coplanar Nozzle, AIAA 2008-2969, 14th AIAA/CEAS Conference.

${ }^{18}$ Eschricht, D., Panek, L., Yan, J., Michel, U. and Thiele, F., Jet Noise Prediction of a Serrated Nozzle, AIAA 2008-2971, 14th AIAA/CEAS Conference.

${ }^{19}$ Li, X.D., Schemel, C., Michel, U. and Thiele, F., Azimuthal Sound Mode Propagation in Axisymmetric Duct Flows, AIAA Journal, Vol. 42, No. 10, 2004, pp. 2019-2027.

${ }^{20}$ Zhang, X., Chen, X.X., Morfey, C.L. and Nelson, P.A., Computation of Spinning Modal Radiation from an Unflanged Duct, AIAA Journal Vol. 42, No. 9, September 2004.

${ }^{21}$ Zhang, X., Chen, X.X. and Morfey, C.L., Acoustic radiation from a semi-infinite duct with a subsonic jet, International Journal of Aeroacoustics Vol. 4, No. 1+2, 2005 pp.169-184. 


\section{Figures}

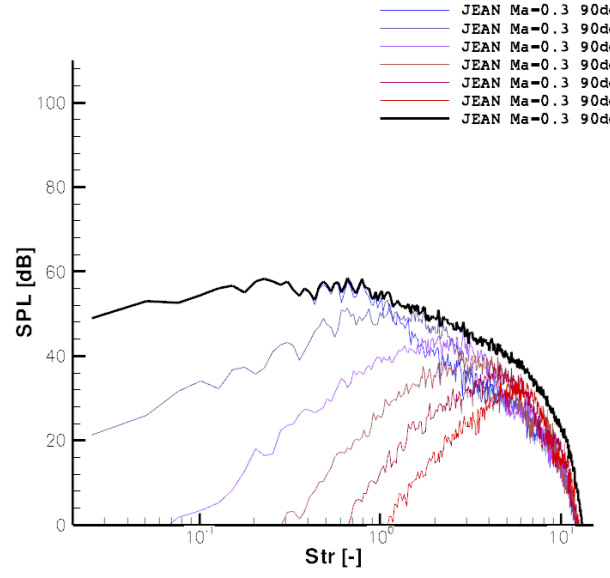

(a) $M a=0.3, \theta=90^{\circ}$

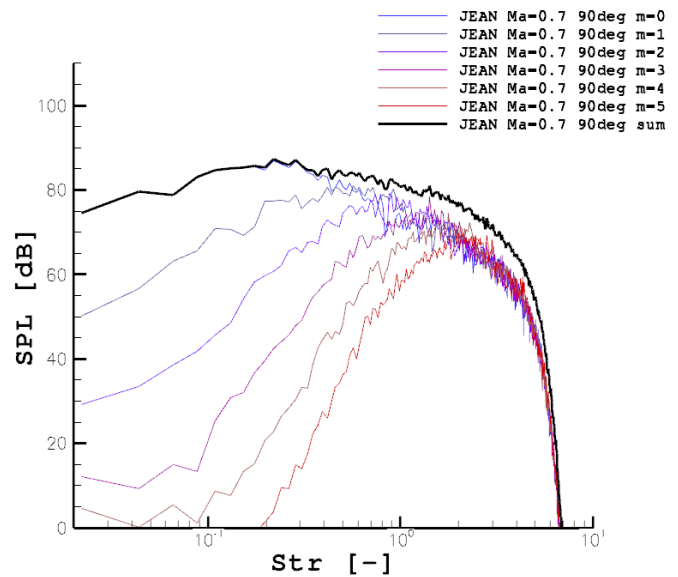

(c) $M a=0.7, \theta=90^{\circ}$

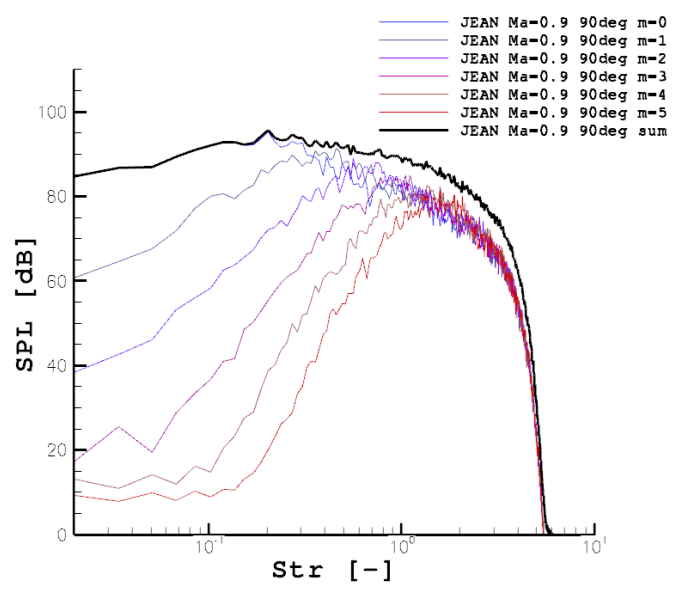

(e) $M a=0.9, \theta=90^{\circ}$

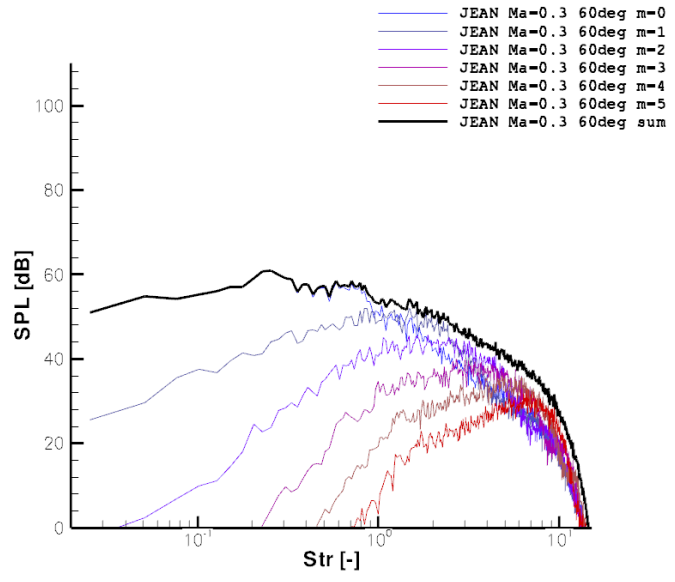

(b) $M a=0.3, \theta=60^{\circ}$

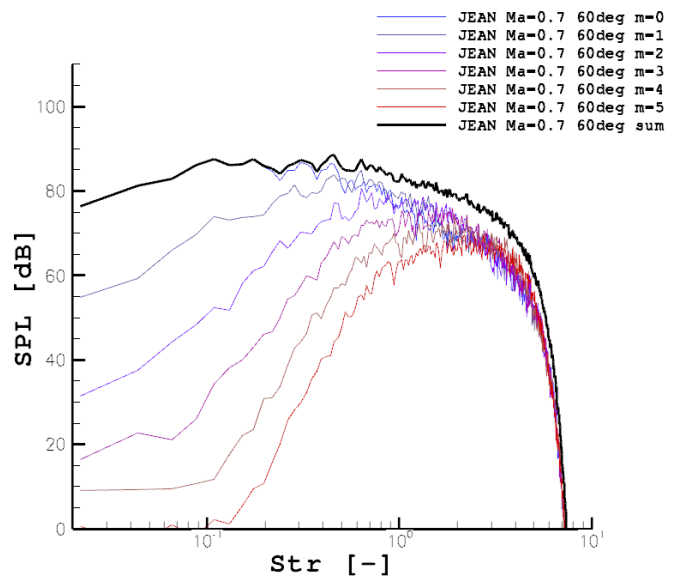

(d) $M a=0.7, \theta=60^{\circ}$

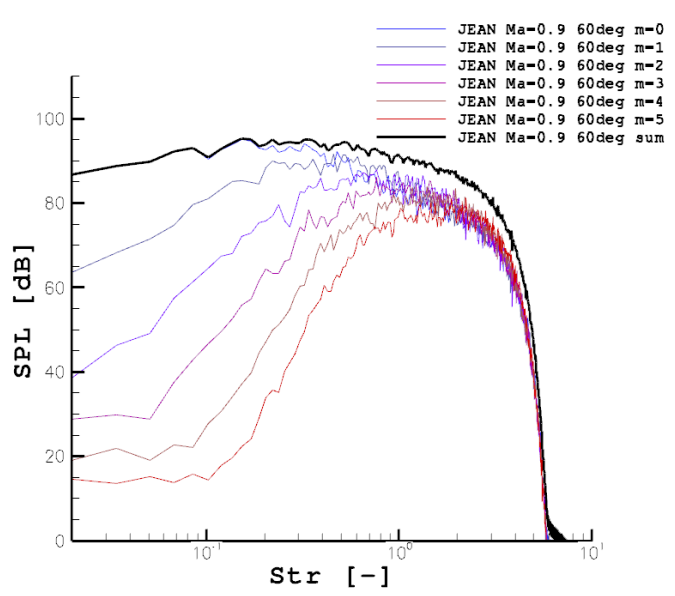

(f) $M a=0.9, \theta=60^{\circ}$

Figure 17. The six independently computed azimuthal mode spectra and their sum for a static cold single stream jet with Mach numbers $M a=0.3,0.7,0.9$ at the polar angles $\theta=90^{\circ}, 60^{\circ}$ 


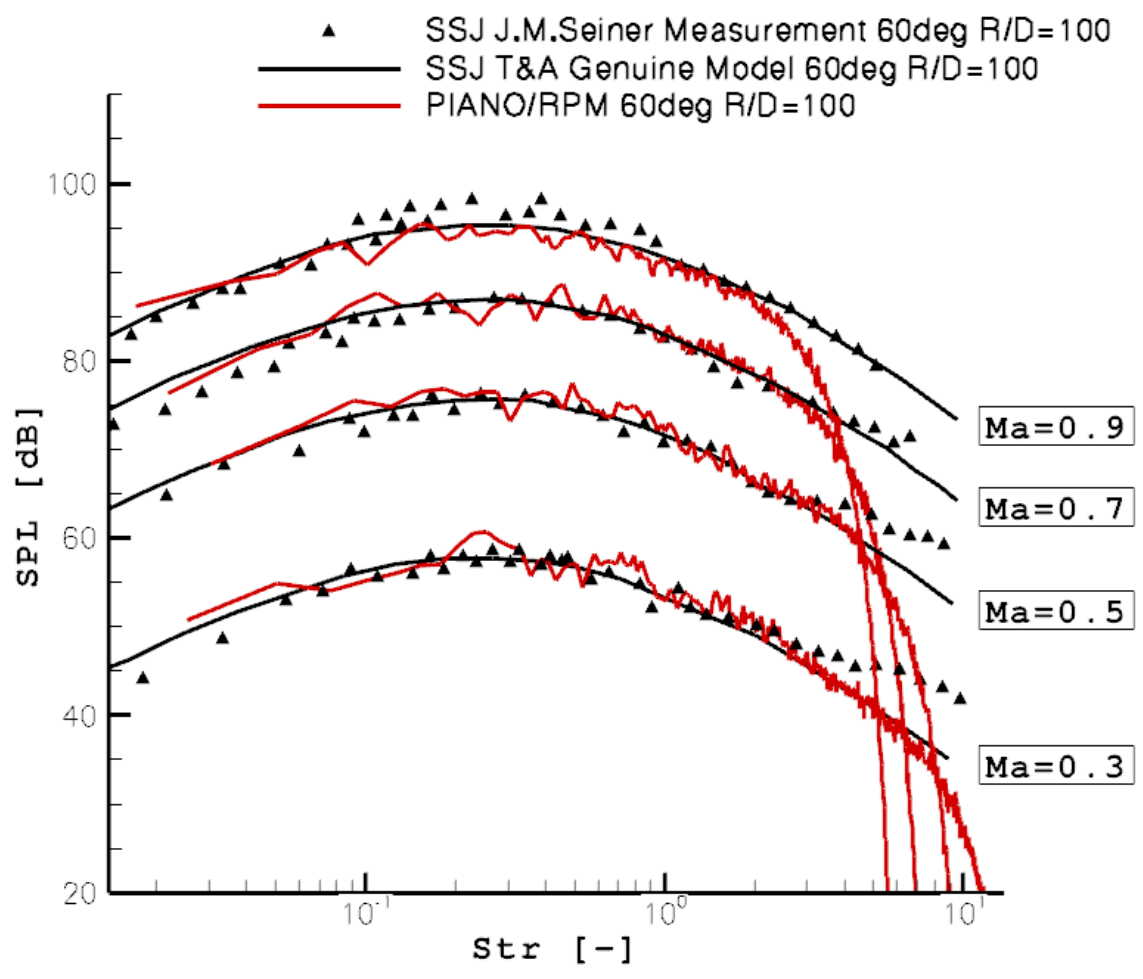

(a) $\theta=60^{\circ}$

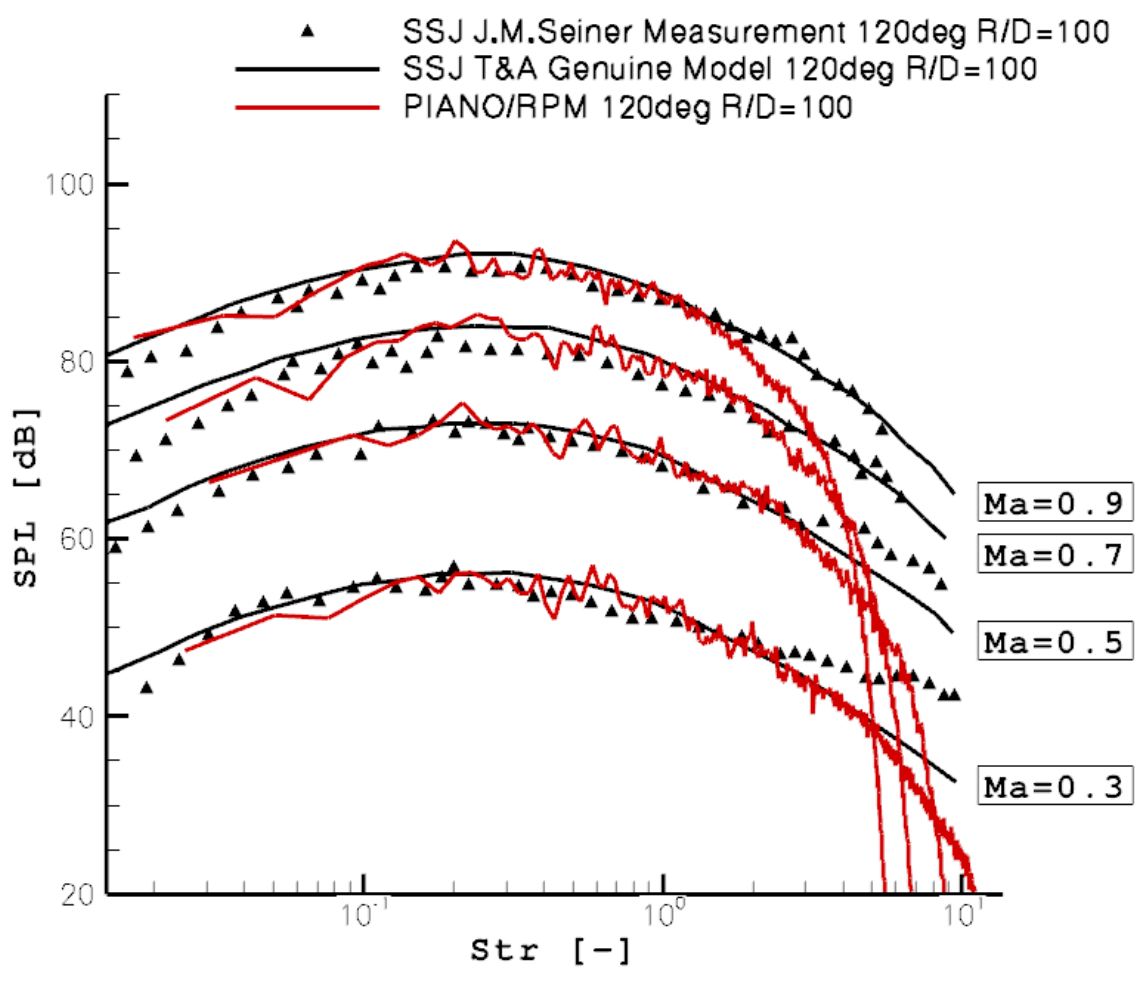

(b) $\theta=120^{\circ}$

Figure 18. Cold single stream jet spectra obtained by PIANO/RPM at the polar angle $\theta=60^{\circ}$ and $\theta=120^{\circ}$ with Mach numbers $M a=0.3,0.5,0.7,0.9$ in comparison to T\&A model and measurements 


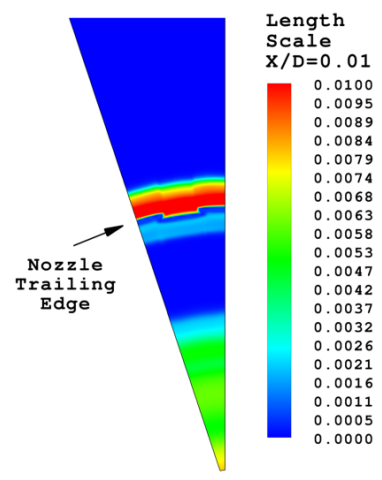

(a.I)

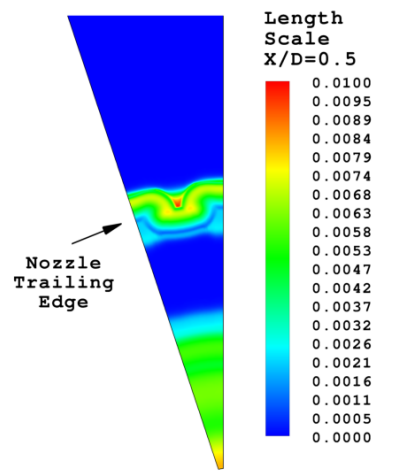

(a.II)

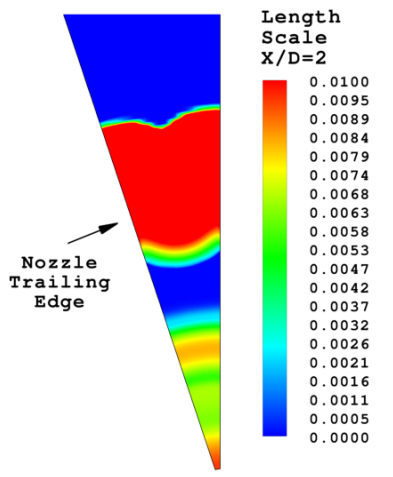

(a.III)

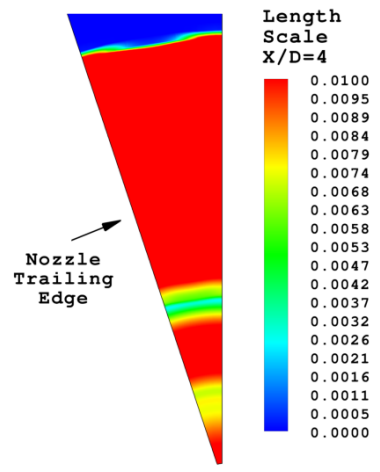

(a.IV)

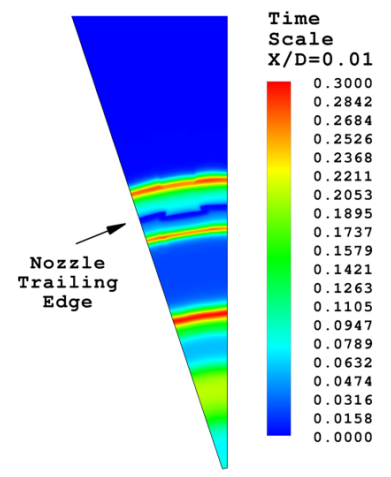

(b.I)

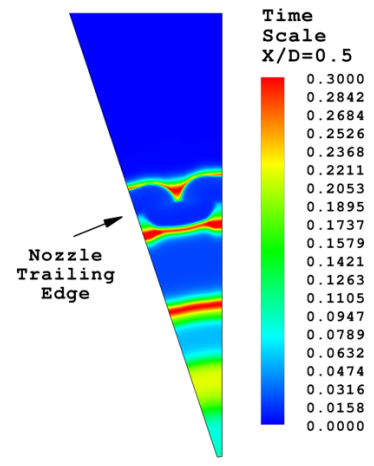

(b.II)

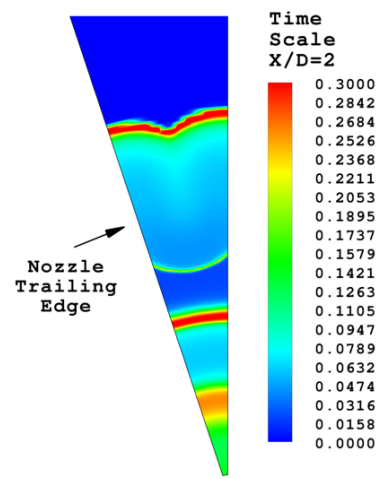

(b.III)

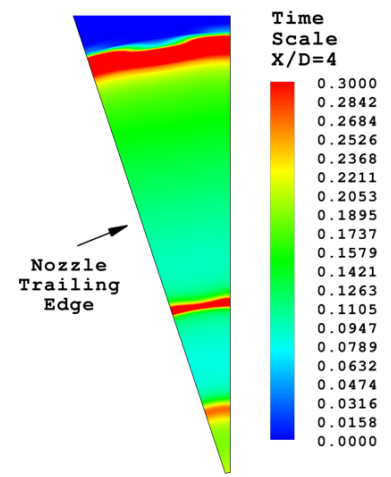

(b.IV)

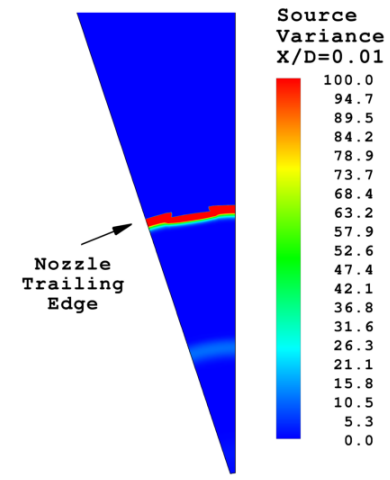

(c.I)

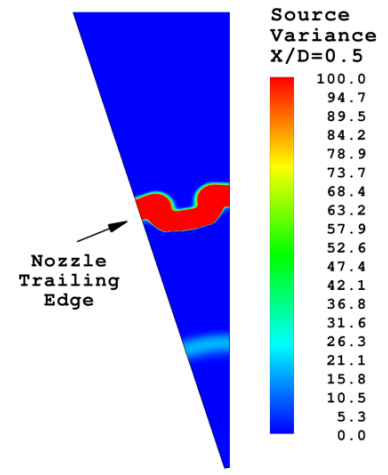

(c.II)

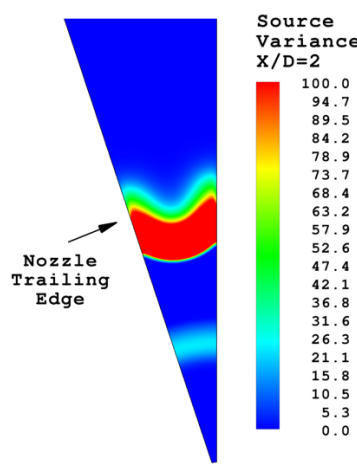

(c.III)

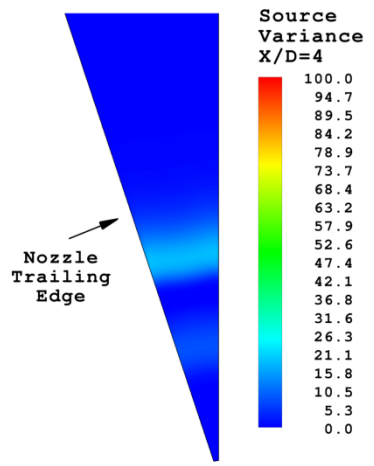

(c.IV)

Figure 19. Statistical quantities of a serrated nozzle extracted from RANS, variation in azimuthal direction at $X / D=0.01,0.5,2.0,4.0$ 


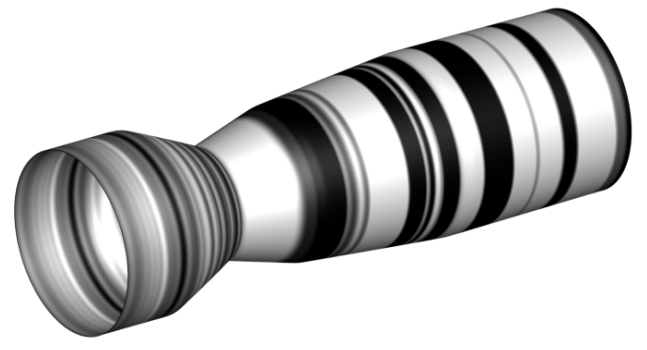

(a) $m=0$

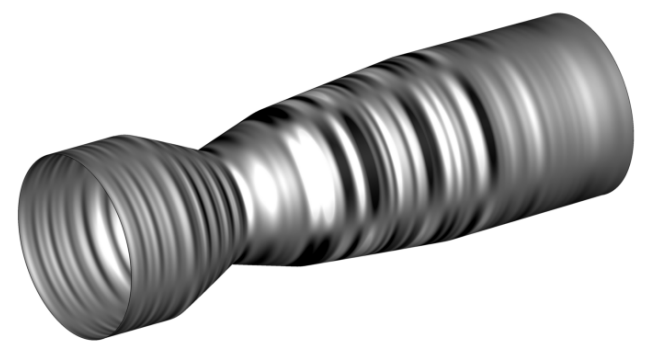

(c) $m=2$

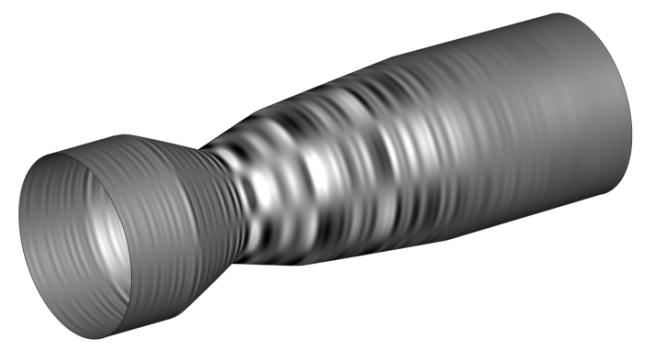

(e) $m=4$

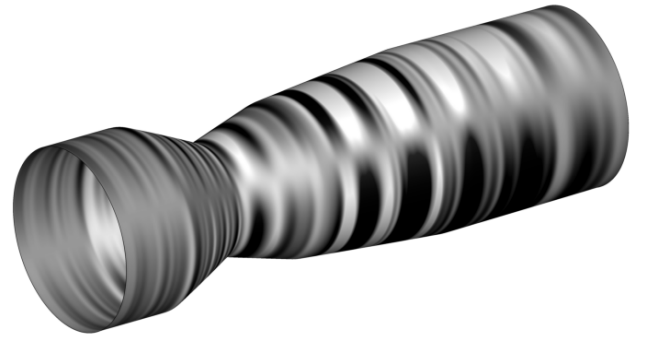

(b) $m=1$

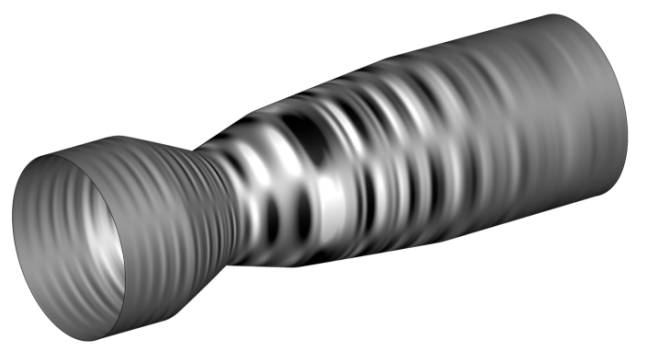

(d) $m=3$

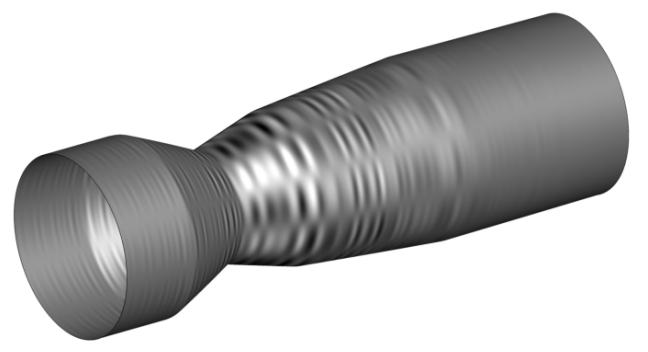

(f) $m=5$

Figure 20. Azimuthal extruded FWH-surface data of a 3-D modal CAA computation for azimuthal modes $m=0 \ldots 5$ (radial distance $R \approx 5.0 \cdot D_{j}$ )

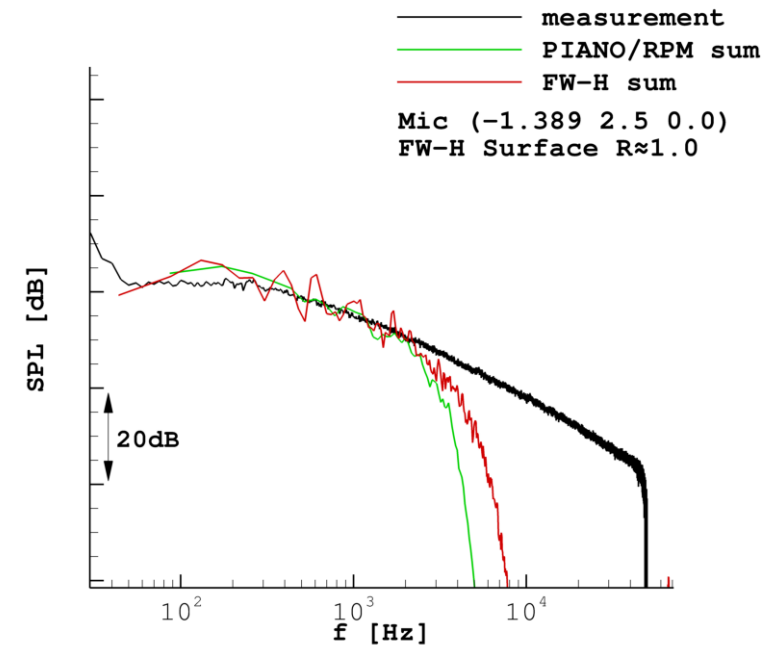

(a) $\theta=120^{\circ}$

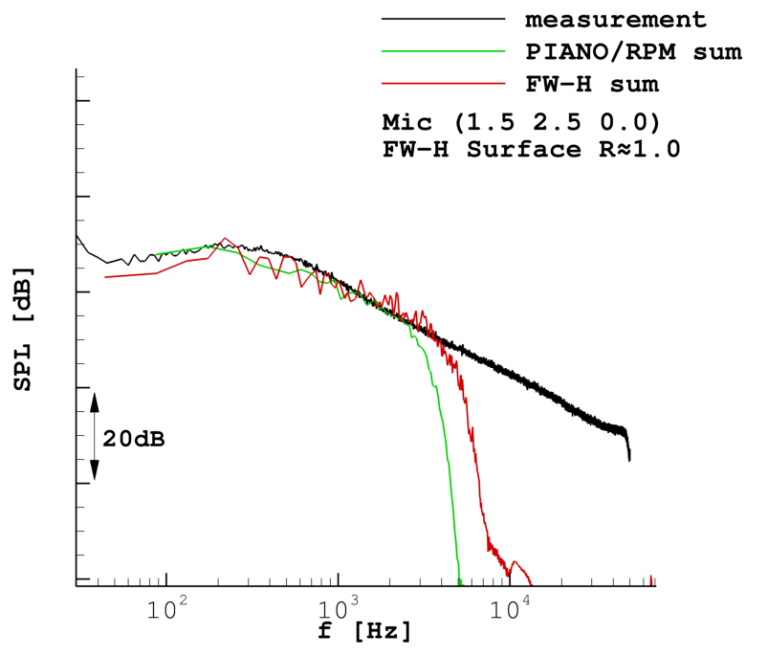

(b) $\theta=60^{\circ}$

Figure 21. Sum spectra of CAA computation and FWH extrapolation with surface at $R \approx 5.0 \cdot D_{j}$ 

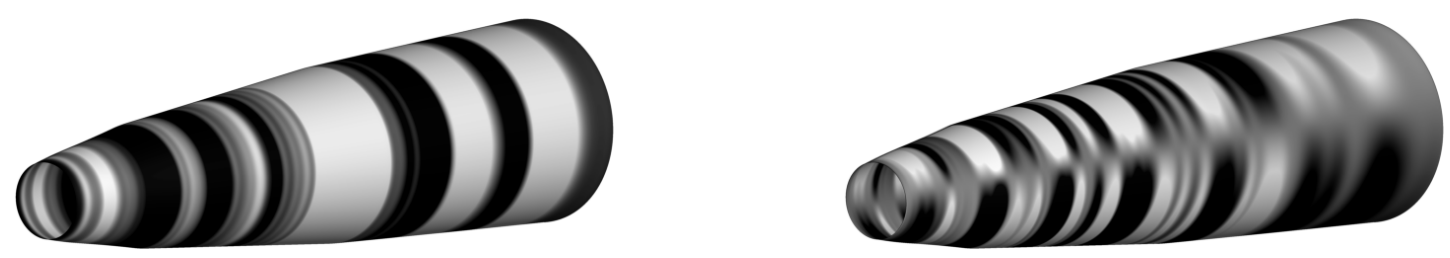

(a) $m=0$

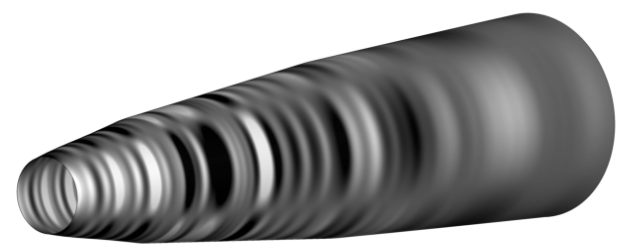

(c) $m=2$

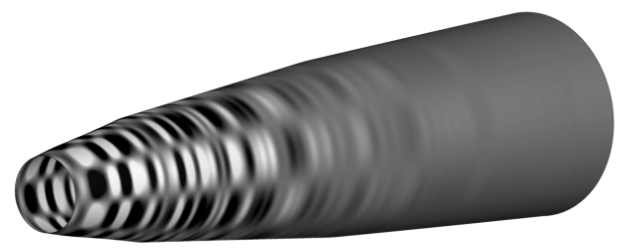

(e) $m=4$ (b) $m=1$

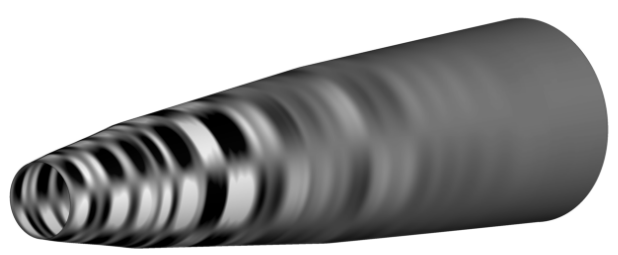

(d) $m=3$

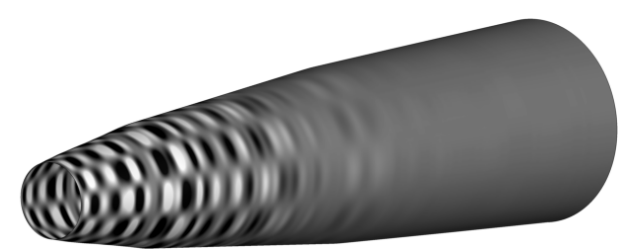

(f) $m=5$

Figure 22. Azimuthal extruded FWH-surface data of a 3-D modal CAA computation for azimuthal modes $m=0 \ldots 5$ (radial distance $R \approx 2.5 \cdot D_{j}$ )

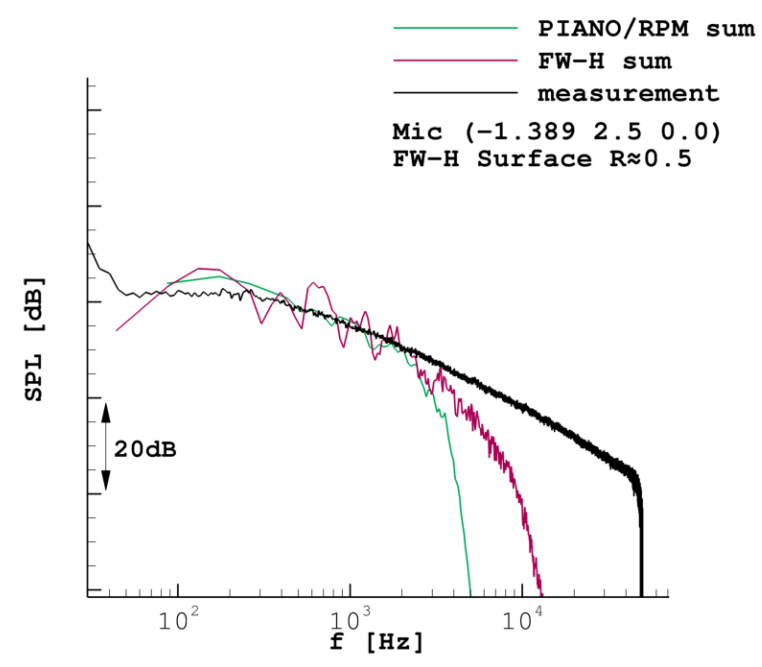

(a) $\theta=120^{\circ}$

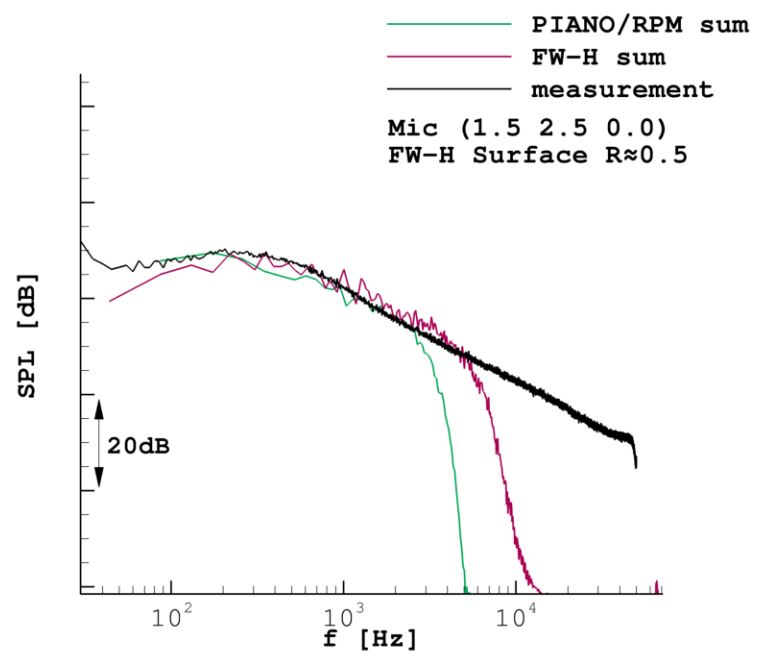

(b) $\theta=60^{\circ}$

Figure 23. Sum spectra of CAA computation and FWH extrapolation with surface at $R \approx 2.5 \cdot D_{j}$ 